\title{
Curcumin promotes progression of AApoAII amyloidosis and peroxisome proliferation in mice by activating the PPAR $\alpha$ signaling pathway
}

\section{Jian Dai ${ }^{1,2}$, Ying $\mathrm{Li}^{3}$, Fuyuki Kametani ${ }^{4}$, Xiaoran Cui ${ }^{5}$, Yuichi Igarashi ${ }^{5}$, Jia Huo ${ }^{6}$, Hiroki Miyahara $^{1,7}$, Masayuki Mori ${ }^{1,7}$, Keiichi Higuchi ${ }^{1,7}$}

${ }^{1}$ Department of Neuro-health Innovation, Institute for Biomedical Sciences, Interdisciplinary Cluster for Cutting Edge Research, Shinshu University, Matsumoto 390-8621, Japan

${ }^{2}$ Department of Pathology, the Xiehe Hospital of Tangshan, Tangshan 063000, China

${ }^{3}$ Aging Biology, Department of Biomedical Engineering, Graduate School of Medicine, Science and Technology Shinshu University, Matsumoto 390-8621, Japan.

${ }^{4}$ Department of Dementia and Higher Brain Function, Tokyo Metropolitan Institute of Medical Science, Tokyo 156-8506, Japan.

${ }^{5}$ Department of Aging Biology, Institute of Pathogenesis and Disease Prevention, Shinshu University Graduate School of Medicine, Matsumoto 390-8621, Japan,

${ }^{6}$ Department of Orthopedic Surgery, the Third Hospital of Hebei Medical University, Shijiazhuang 050030, China

${ }^{7}$ Department of Aging Biology, Shinshu University School of Medicine, Matsumoto 390-8621, Japan

\begin{abstract}
:
Curcumin is a polyphenol compound that exhibits multiple physiological activities. To elucidate the mechanisms by which curcumin affects systemic amyloidosis, we investigated amyloid deposition and molecular changes in a mouse model of amyloid apolipoprotein A-II (AApoAII) amyloidosis, in which mice were fed a curcumin-supplemented diet. Curcumin supplementation for 12 weeks significantly increased AApoAII amyloid deposition relative to controls, especially in the liver and spleen. Liver weights and plasma ApoA-II and high-density lipoprotein concentrations were significantly elevated in curcumin-supplemented groups. RNA-sequence analysis revealed that curcumin intake affected hepatic lipid metabolism via the peroxisome proliferator-activated receptor (PPAR) pathway, especially PPAR $\alpha$ activation, resulting in increased Apoa 2 mRNA expression. The increase in liver weights was due to activation of PPAR $\alpha$ and peroxisome proliferation. Taken together, these results demonstrate that curcumin is a PPAR $\alpha$ activator and may affect expression levels of proteins involved in amyloid deposition to influence amyloidosis and metabolism in a complex manner.
\end{abstract}




\section{Introduction :}

2 Amyloidosis is a group of diseases characterized by abnormal aggregation of proteins to form

3 amyloid fibrils, and subsequent deposition in various tissues and organs, which can lead to severe functional failures. More than 30 amyloid proteins have been identified; some result in localized tissue deposits, such as $\mathrm{A} \beta$ in Alzheimer's disease and $\alpha$-synuclein ( $\alpha \mathrm{Syn})$ in Parkinson's disease that deposit in the brain, while others result in systemic amyloidosis and are widely deposited in various tissues and organs, such as AL $\lambda$ and AL $\kappa$ in immunoglobulin light chain amyloidosis and ATTR in transthyretin amyloidosis [1]. In general, when amyloid proteins are exposed to certain conditions that affect protein homeostasis (e.g., overexpression, gene mutation, enzyme cleavage), they may undergo structural changes into stable structures that are rich in $\beta$-sheets, and which promote subsequent aggregation to form oligomers, protofibrils and amyloid fibrils [2,3]. Because the formation of amyloid fibrils is nearly irreversible, maintaining proteostasis and inhibiting amyloid aggregation presents a challenge for development of an effective treatment.

Some natural phenolic compounds extracted from plants exhibit certain anti-amyloid activity in vitro and in vivo [4]. Curcumin, a polyphenol compound, is extracted from the rhizome of Curcuma longa and has a long history of use in traditional medicines in some countries in Asia. In in vitro experiments, curcumin has been shown to suppress the aggregation and cytotoxicity of A $\beta, \alpha$ Syn, islet amyloid precursor protein (IAPP), ATTR and prion protein (PrP) [4]. In 2001, the first evidence of the efficacy of curcumin against $A \beta$ amyloidosis in a transgenic model mice was reported [5]. Curcumin was found to suppress amyloid deposition in a mouse model of Alzheimer's disease and improve memory function. It was subsequently demonstrated that the amount of amyloid present in TTR- and tau-transgenic mice were reduced by curcumin supplementation [6-8]. Due to the strong affinity of curcumin for the amyloid structure, it is believed that curcumin inhibits the formation of amyloid fibrils by binding to amyloid protein monomers or aggregates $[4,5,9,10]$. This curcumin-protein complex exhibits better stability and reduces the tendency to aggregate. However, another mechanism that has been proposed suggests that curcumin inhibits $A \beta$ production by down-regulating the expression of amyloid-beta precursor protein (APP) or beta-site APP cleaving enzyme 1 (BACE1) in vitro [11, 12]. Unfortunately, there are few reports that suggest that curcumin will provide clinical benefit in patients with Alzheimer's disease or AL amyloidosis [4, 13, 14]. In fact, it is unclear how curcumin inhibits amyloid deposition in vivo. 
1 anti-inflammatory, anti-cancer, lipid metabolism regulation and anti-amyloid properties.

2 However, a link between the various physiological activities has not been completely

3 established [15]. Curcumin has been found to exert an influence on multiple signaling pathways

4 [16]. In 2003, curcumin was first shown to inhibit rat hepatic stellate cell growth by activation

5 of PPAR $\gamma$, suggesting that curcumin might have an effect on the PPAR signaling pathway [17].

6 In mammals, the PPAR subfamily (PPARs) is a group of nuclear receptor proteins, e.g.,

7 transcription factors, and consists of three members, namely PPAR- $\alpha$, PPAR- $\beta / \delta$ and PPAR $-\gamma$,

8 that play essential roles in the regulation of metabolic homeostasis, glucose and energy

9 metabolism, cellular differentiation, inflammation, and ROS metabolism [18-21]. The functions

10 of the three PPAR subtypes are different. PPAR- $\alpha$ regulates fatty acid transport and oxidative

11 decomposition in the liver and muscle in response to energy metabolism levels. PPAR $\gamma$ mainly

12 regulates fatty acid synthesis and fat accumulation in adipose tissue, as well as differentiation of

13 adipose cells and macrophages. PPAR $\beta / \delta$ plays an important role in lipid catabolism, energy

14 homeostasis and cell differentiation, but the mechanism and network of action are not

15 completely clear [21, 22]. Among the three isotypes, the relationship between curcumin and

$16 \operatorname{PPAR} \gamma$ is the most extensively studied, while information on $\alpha$ and $\beta / \delta$ remains scarce.

In this study, we sought to determine whether curcumin affects the amyloid deposition process besides directly binding to amyloid proteins, and identify a link between curcumin's anti-amyloid activity and its various other biological activities. In our previous study, we found that antioxidants (tempol and apocynin) can effectively reduce AApoAII amyloid deposition [23]. It is therefore possible that the anti-oxidative effects of curcumin also play an important role in amyloid formation. We examined the effects of curcumin supplementation in a mouse model of AApoAII amyloidosis, in which mice were induced to develop systemic amyloidosis [24]. In contrast to expectations, our results showed that curcumin significantly promoted AApoAII amyloid deposition by activating the PPAR signaling pathway. Moreover, our results suggest that activation of PPAR $\alpha$ plays a major role in the amyloid formation process. 


\section{Results :}

2 Degree of AApoAII amyloid deposition and liver weights were significantly increased after 3 supplementation with curcumin.

4 Two-month-old female R1.P1-Apoa $2^{c}$ mice were divided into 4 groups: the control (Con) and curcumin (Cur) groups are non-amyloid-induced groups and fed a common diet or $2 \% \mathrm{w} / \mathrm{w}$ curcumin diet, respectively. The other two groups were injected with $1 \mu \mathrm{g}$ AApoAII amyloid fibrils into the tail vein to induce amyloidosis, and were fed a common diet (A-NT group) or 2\% curcumin diet (A-Cur group) (see experimental design in Supplementary Fig. 1). After 8 and 12 weeks, we evaluated the effect of curcumin intake on amyloid deposition. Unlike previous studies, the degree of amyloid deposition in the A-Cur group was significantly increased compared to the A-NT group at both 8 and 12 weeks, especially in the liver and spleen (Fig. 1a-d, Supplementary Fig. 2). This is the first evidence that curcumin promotes amyloid deposition in vivo, and suggests that curcumin can affect the amyloid deposition process via a complex mechanism, not just via binding with amyloid protein monomers or aggregates. In addition, no amyloid deposition was observed in the Cur group, suggesting that curcumin does result in the pathogenesis of amyloidosis without induction by amyloid fibril injection. These results suggest that curcumin accelerates amyloid deposition but does not cause structural changes in amyloidogenic proteins.

On the other hand, we noticed that the livers of mice were larger and heavier in those mice that received dietary supplementation with curcumin than in those without supplementation (Fig. 1 e-f, Supplementary Table 1). In the subsequent histological observation, it was found that the mice in the curcumin-supplemented groups had hepatocyte hypertrophy and abnormal changes in some hepatocyte nuclei, but no tumors or abnormal organisms were observed (Supplementary Fig. 3). This is somewhat confusing, as it has been demonstrated in many high-fat diet experiments that curcumin effectively reduces liver lipid deposition and liver weight $[25,26]$. To exclude the effects of hepatocyte damage or inflammation, plasma levels of aspartate aminotransferase (AST) and alanine transaminase (ALT) and several inflammatory marker cytokines in the liver were detected (Supplementary Figs. 4 and 5). However, we found no support that dietary supplementation with curcumin causes inflammation or cell injury in the liver. To confirm whether different doses will affect the experimental results, we repeated the experiment with a lower dose $(0.5 \% \mathrm{w} / \mathrm{w})$ of curcumin diet and obtained similar changes in the degree of amyloid deposition and liver weight (Supplementary Fig. 6). 
bioRxiv preprint doi: https://doi org/10.1101/2020.09.23.309302; this version posted September 23, 2020. The copyright holder for this preprint (which was not certified by peer review) is the author/funder, who has granted bioRxiv a license to display the preprint in perpetuity. It is made available under aCC-BY-NC-ND 4.0 International license.

\section{Figure 1}

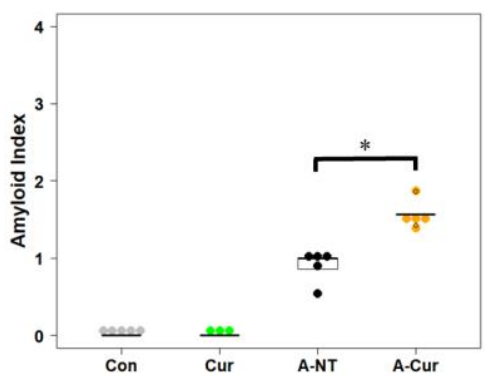

2

C

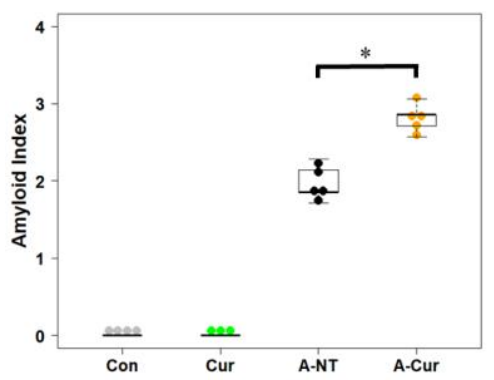

3

4

6

8

e

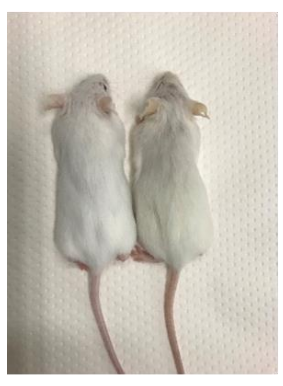

f

8-week group b

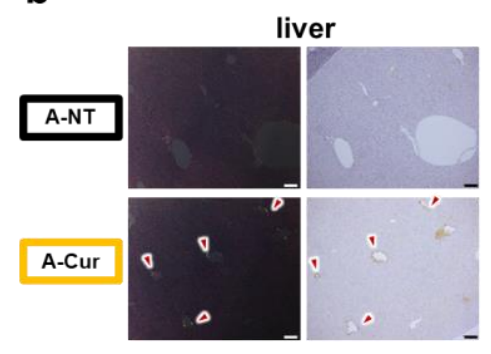

d
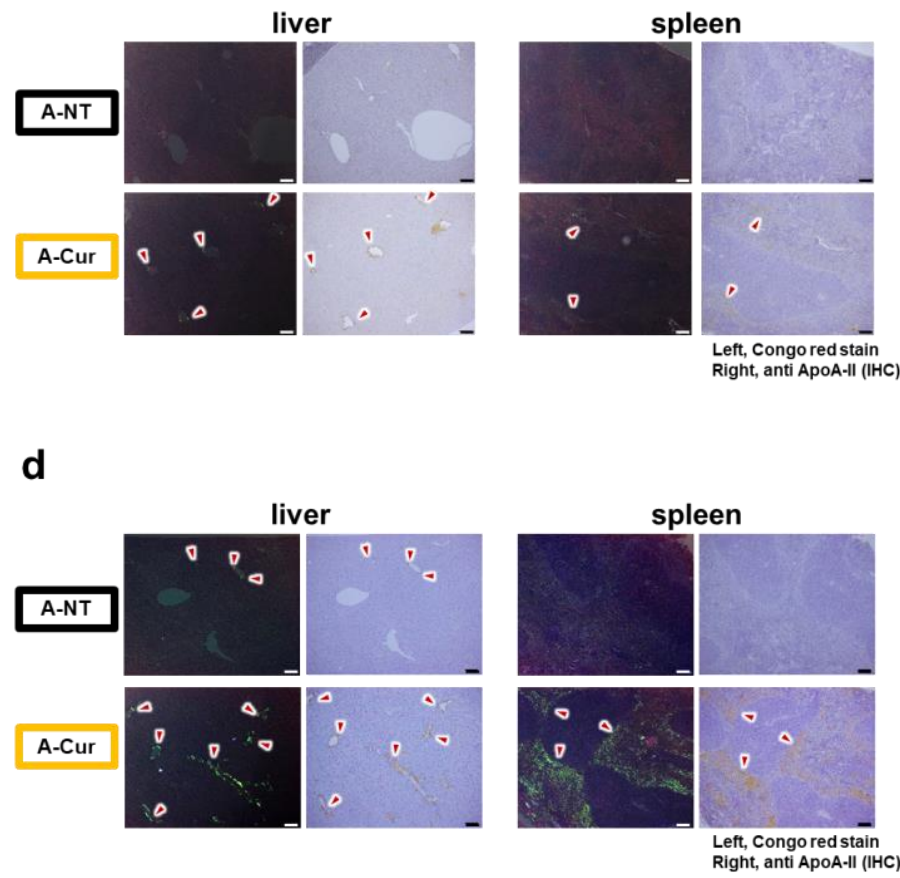
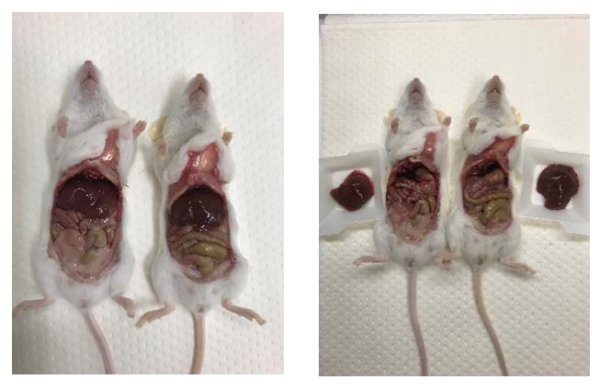

Left: commercial diet

12-week group
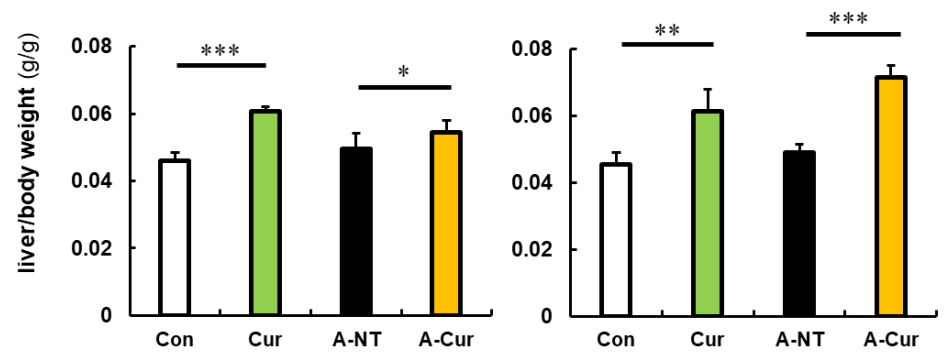

Figure 1. Degree of AApoAII amyloid deposition and liver weights

(a) Amyloid index (AI) in the 8-week group. (b) Representative Congo red and IHC images of AApoAII amyloid deposition in the 8-week group. Amyloid deposits (red arrows) were identified by green birefringence in Congo red-stained sections using polarizing light microscopy. Each scale bar indicates $100 \mu \mathrm{m}$. (c) Amyloid index in the 12-week group. (d) Representative Congo red and IHC images of amyloid deposition in the 12-week group. (e) Mice in curcumin-supplemented group have larger livers and less adipose tissue in the abdominal cavity than mice without curcumin supplementation (left: commercial diet; right: curcumin diet) (f) Ratio of liver weight / body weight in all groups. Each dot represents an individual mouse (a, c). Data are mean \pm SD (f). $N=3-5$. The Kruskal-Wallis test with the Steel-Dwass test was used for the amyloid index, and the Tukey-Kramer method was used for multiple comparisons of liver weights; ${ }^{*} \mathrm{P}<0.05,{ }^{*} \mathrm{P}<0.01,{ }^{*} * * \mathrm{P}<0.001$. 


\section{Curcumin elevated levels of ApoA-II protein and affected lipid metabolism in mice.}

2 It has been reported that overexpression of amyloid protein or precursor protein is one of the most important factors contributing to the pathogenesis of amyloidosis, and results in increased amyloid deposition in transgenic model mice [2, 3, 27]. Apolipoprotein A-I (ApoA-I) and apolipoprotein A-II (ApoA-II) are the major proteins comprising HDL (high-density lipoprotein) particles, and curcumin has been shown to increase HDL levels in some studies of lipid metabolism [28, 29]. We previously demonstrated that overexpression of ApoA-II can

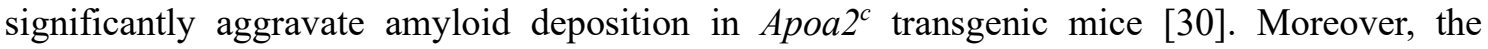
co-deposited proteins in AApoAII amyloidosis may also affect the degree of amyloid deposition [31]. We hypothesized that the increase in amyloid deposition in the curcumin diet group was due to upregulation of ApoA-II or other co-deposition proteins. Plasma levels of ApoA-II and the major proteins that were co-deposited with AApoA-II amyloids (ApoA-I and ApoE) were measured by Western immunoblot. In line with our expectations, significantly higher plasma levels of ApoA-II at 12 weeks were observed for the Cur group compared with the Con group (Fig. 2a). Following the increase in amyloid deposition, the plasma levels of ApoA-II will gradually decrease upon tissue deposition [31]. As shown in Fig. 2a, plasma levels of ApoA-II in the A-Cur group decreased significantly compared with those in the Cur group, while the decline in the A-NT group was mild compared with that in the Con group. These different degrees of decline resulted in a narrowing difference between the A-NT and A-Cur groups. ApoA-I plasma levels showed a slight increase with curcumin supplementation, but were not significant (Fig. 2b). Consistent with previous results [23], ApoE, which is the most abundant co-deposited protein in AApoAII amyloidosis, was significantly increased in the amyloid-induced groups, but was not significantly affected by curcumin (Fig. 2c).

Because ApoA-II, ApoA-I, and ApoE are all constituent proteins of HDL and are related to lipid metabolism, we evaluated the change in lipid metabolism by detecting plasma levels of total cholesterol, HDL cholesterol and triglycerides. Results suggest that curcumin supplementation increased HDL cholesterol levels, but reduced triglycerides levels (Fig. 2e-g), which is consistent with previous experiments of curcumin supplementation. Similar results were also shown in lipid metabolism studies, in which hypolipidemic agents, namely fibrates, were used as PPAR $\alpha$ agonists $[32,33]$.

Further, immunoblotting results of ApoA-I and ApoE in the 8-week group are consistent with those of the 12-week group, but the change in ApoA-II protein did not show a significant difference. These results indicate that the increase in ApoAII levels may have undergone a slow process to adapt to the changes in lipid metabolism (Supplementary Fig. 7). 
a
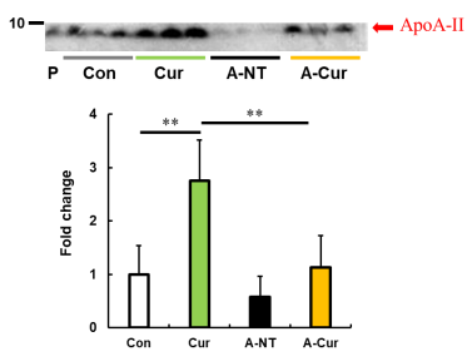

b

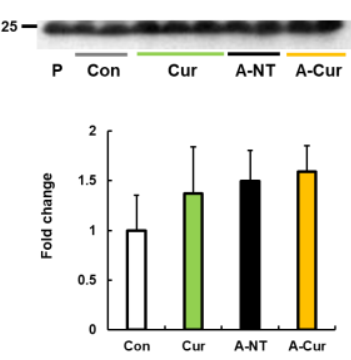

c
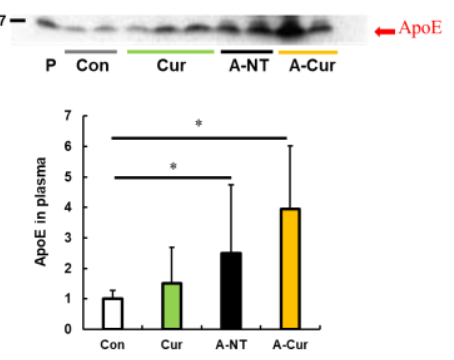

d

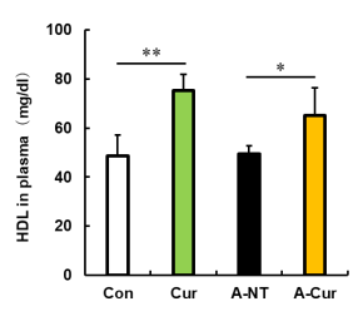

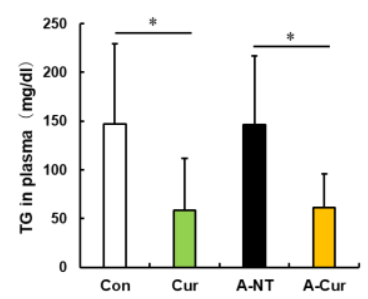

f

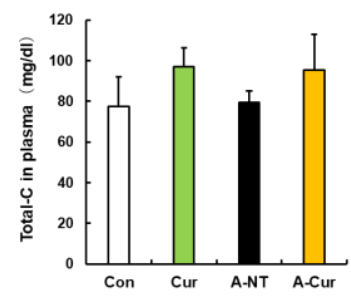

Figure 2. Curcumin elevated ApoA-II levels and affected lipid metabolism in mice after 12 weeks. (a-c) Plasma concentrations of ApoA-II, ApoA-I, and ApoE were determined by densitometry of Western immunoblot after SDS-PAGE. Representative results of Western blot are shown above the figures. Histograms show fold changes relative to the Con group and represent the means \pm SD. P indicates the pooled plasma of female R1.P1-Apoa2c mice at 2 months of age $(\mathrm{N}=4)$ that did not have AApoAII amyloid deposits, as the positive control. (d-f) Plasma concentrations of HDL cholesterol, triglycerides (TG) and total cholesterol (Total-C) were determined using quantitative assay kits. $\mathrm{N}=3-5$. The Tukey-Kramer method was used for multiple comparisons; $* \mathrm{P}<0.05 . * * \mathrm{P}<0.05$.

4

RNA sequence analysis showed that curcumin regulates many lipid metabolism-related genes via the PPAR signaling pathway, especially by activating PPAR $\alpha$ in the liver.

Although it has been suggested that curcumin inhibits amyloid fibril formation by binding to amyloid proteins and maintaining protein homeostasis, our results showed that curcumin may also affect the degree of amyloid deposition by other means. Several physiological activities of curcumin are related to the activation of $\operatorname{PPAR} \gamma$, including decreased insulin resistance, anti-inflammatory, and anti-cancer activities [15, 16, 34, 35]. However, ApoAII expression is thought to be upregulated upon activation of PPAR $\alpha$ to enhance the delivery of lipids from the periphery tissue to the liver [36, 37]. To identify possible signaling pathways or target proteins that respond to curcumin, we performed a comprehensive analysis of mRNA transcription in the liver using the RNA sequence method. As shown in the Venn diagram (Fig. 3a), 75 genes are changed in mRNA expression by curcumin supplementation regardless of induction of amyloidosis (Supplementary Table 2). The enrichment pathway analysis based on the KEGG database suggested that differentially expressed genes (DEGs) are mainly distributed in lipid metabolism-related signaling pathways centered on the PPAR pathway (Fig. 3b). Analysis of the 
1 non-amyloid induced groups separately showed that 98 genes were upregulated and 93 genes were down-regulated in the Cur group compared with the Con group. Among the 15 genes that were most significantly upregulated, more than $2 / 3$ were related to fatty acid transport and fatty

\section{Figure 3}

a

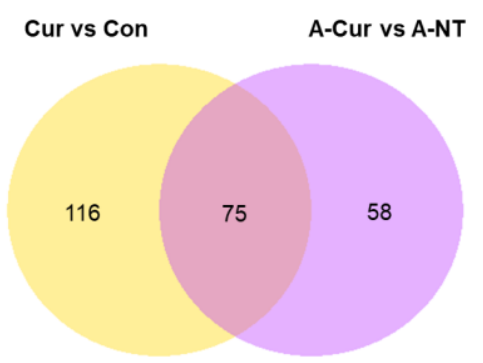

13

14

16

17

18

19

20

21

22

C Curvs Con

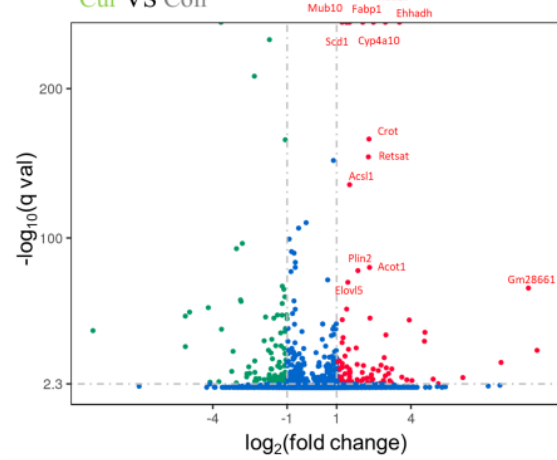

b

\begin{tabular}{|c|c|c|c|}
\hline Rank & Term & $\begin{array}{l}\text { Sample numberl } \\
\text { Background }\end{array}$ & Corrected P-value \\
\hline 1 & $\begin{array}{l}\text { PPAR signaling } \\
\text { pathway }\end{array}$ & $17 / 82$ & $1.22 \mathrm{e}-10$ \\
\hline 2 & Retinol metabolism & $17 / 88$ & $1.65 \mathrm{e}-10$ \\
\hline 3 & Fatty acid degradation & $10 / 49$ & $2.04 \mathrm{e}-06$ \\
\hline 4 & Metabolic pathways & $50 / 1256$ & $2.04 \mathrm{e}-06$ \\
\hline 5 & Chemical carcinogenesis & $12 / 92$ & $5.92 \mathrm{e}-06$ \\
\hline 6 & $\begin{array}{l}\text { Steroid hormone } \\
\text { biosynthesis }\end{array}$ & $11 / 86$ & $1.92 \mathrm{e}-05$ \\
\hline 7 & $\begin{array}{l}\text { Biosynthesis of } \\
\text { unsaturated fatty acids }\end{array}$ & $6 / 25$ & $2.57 \mathrm{e}-04$ \\
\hline 8 & peroxisome & $9 / 81$ & $4.16 \mathrm{e}-04$ \\
\hline$\ldots$ & $\ldots$ & $\ldots$ & $\ldots$ \\
\hline
\end{tabular}

d

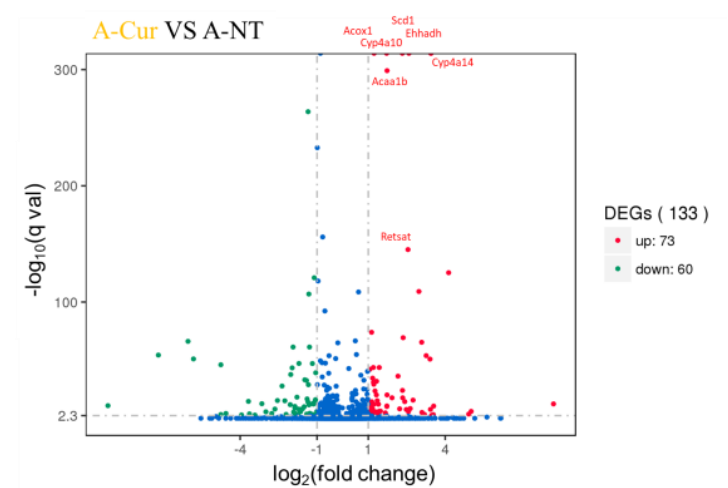

e

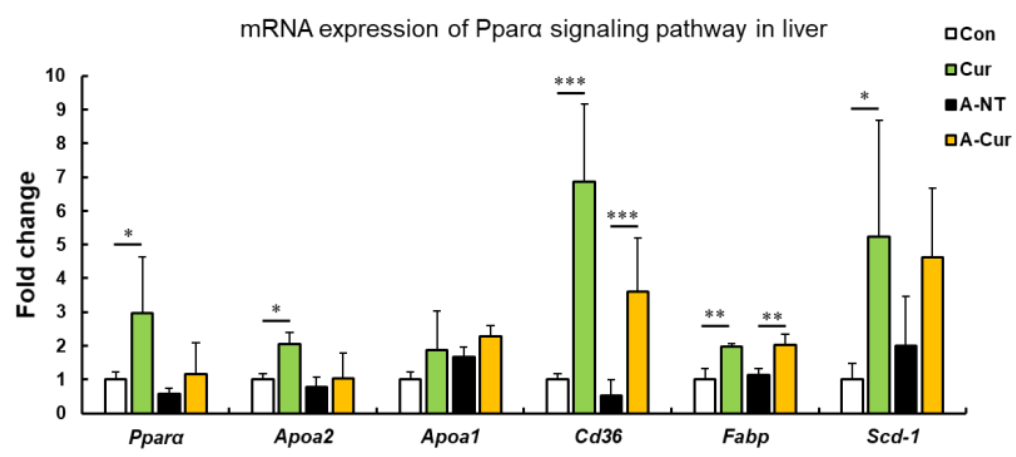

Figure 3. RNA sequence analysis showed that curcumin regulates many lipid metabolism-related genes via the PPAR signaling pathway.

(a) The Venn diagram shows that 75 genes are changed in the liver by supplementation with curcumin for 12 weeks. (b) Enrichment pathway analysis based on the KEGG database. (c-d) The Volcano plot diagram shows that DEGs affected by curcumin are related to lipid metabolism and the PPARa pathway. (e) Regulated genes were identified by real-time qPCR. Histograms show fold changes relative to the Con group. Data are mean \pm $\mathrm{SD}$. The Tukey-Kramer method was used for multiple comparisons of gene changes; $* \mathrm{P}<0.05, * * \mathrm{P}<0.01,{ }^{*} * \mathrm{P}$ $<0.001$. 
1 acid oxidation (Fig. 3c). Even considering the possible interference of AApoAII amyloidosis on gene expression in amyloid-induced groups, most of these genes had still been promoted in the

3 A-Cur group (Fig. 3d).

4 As a transcription factor, PPAR $\alpha$ is a major regulator of lipid metabolism in the liver. When the body is in an energy-deprived state, activated PPAR $\alpha$ can mobilize fatty acids to the liver and promote fatty acid $\beta$ oxidation to produce energy by upregulation of genes involved in fatty acid transport, fatty acid binding, and peroxisomal and mitochondrial fatty acid $\beta$-oxidation [18-20]. During lipid metabolism, the physiological function of PPAR $\gamma$ mainly involves synthesis and elongation of fatty acids and the differentiation of adipocytes for energy storage [19, 20, 34]. Upon analysis of the DEGs (Supplementary Table 2), we found that most of the genes were related to fatty acid oxidation, which suggests that curcumin regulates gene transcription by activated PPAR $\alpha$ in the liver.

13 We further confirmed the elevated mRNA expression levels of Ppara, Apoa2, Apoa1 and some genes related to fatty acid metabolism in curcumin-supplemented groups by real-time qPCR (Fig. 3e). Although Ppary mRNA expression was confirmed to be upregulated by curcumin (Supplementary Fig. 8), its levels were much lower than Ppara in the liver (data not shown). These results suggest that curcumin is a PPAR $\alpha / \gamma$ dual activator, and that the various physiological activities of curcumin may be derived from the complex regulation of PPAR $\alpha$ and PPAR $\gamma$. Because expression of PPAR $\alpha$ and PPAR $\gamma$ varies largely in different tissues and organs, curcumin exhibits diverse physiological activities in different studies depending on the organs that are evaluated [19-21], while Ppara should be the major target of curcumin owing to its abundance in mouse liver.

PPARa levels in the liver were increased and showed more intranuclear localization in mice supplemented with curcumin.

As a transcription factor, activated PPAR $\alpha$ transferred to the nucleus and formed heterodimers with retinoid X receptor (RXR). The heterodimers bind to the peroxisome proliferator response element (PPRE), a specific DNA sequence present in the promoter region of PPAR-regulated genes [17]. To elucidate how curcumin affects PPAR $\alpha$, we evaluated intracellular localization and PPAR $\alpha$ levels in the liver. Compared with mice without curcumin supplementation, the fluorescence signal of PPAR $\alpha$ in the Cur and A-Cur groups was concentrated in the nucleus, resulting in a higher signal intensity (Fig. 4a). Moreover, as shown in Fig. 4b, PPAR $\alpha$ protein levels were increased in curcumin-supplemented mice, which is consistent with real-time qPCR results. These results confirm that the synthesis and activation of PPAR $\alpha$ are promoted by curcumin and also explain the DEGs related to lipid metabolism in the liver. 


\section{Figure 4}

a
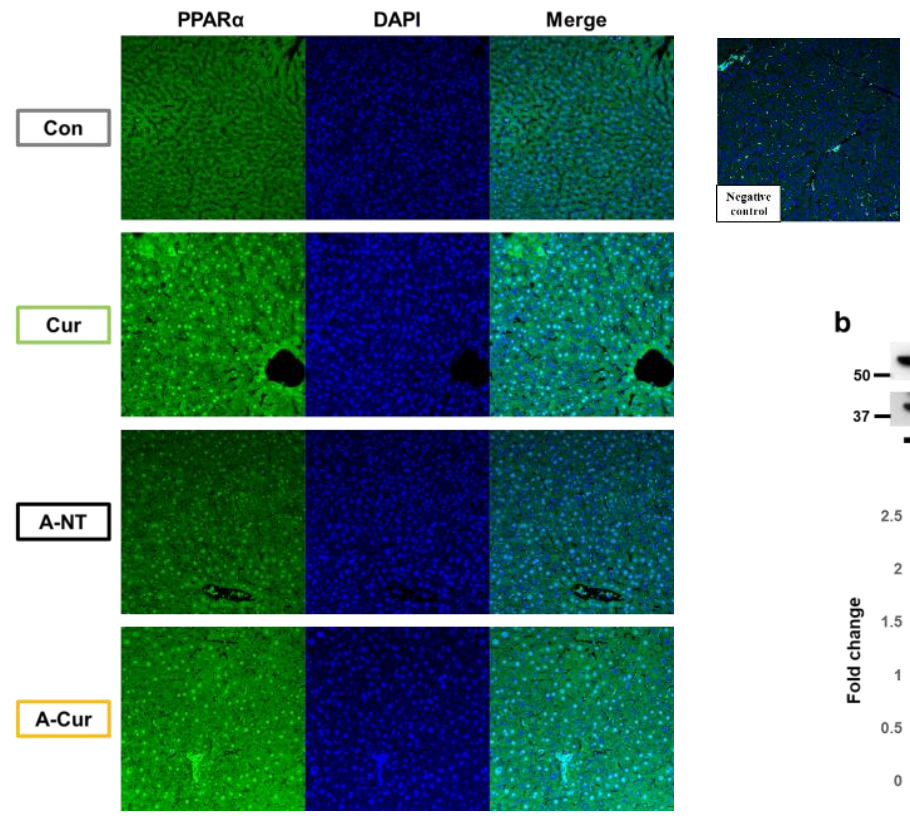

b

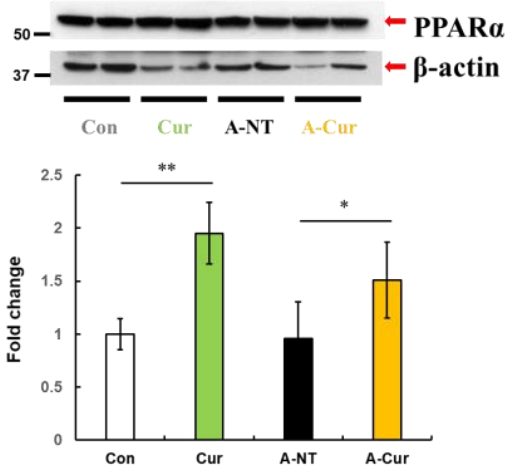

Figure 4. PPARa levels in the liver are increased and show more intranuclear localization in curcumin-supplemented mice.

(a) Immunofluorescence results of PPARa showed obvious intranuclear signal accumulation and extranuclear signal enhancement after curcumin ingestion. (b) Levels of PPARa proteins in the liver were determined by Western immunoblot. Data are mean $\pm \mathrm{SD}$. The Tukey-Kramer method was used for multiple comparisons; $* \mathrm{P}<$ $0.05, * * \mathrm{P}<0.01$.

Curcumin induced a higher abundance of peroxisomes and elevated a variety of peroxisome proteins downstream of PPAR $\alpha$.

Proliferation of peroxisomes provides additional evidence that PPAR $\alpha$ is a target protein of curcumin. The mitochondria and peroxisomes are the most important organelles responsible for fatty acid oxidation. However, very long chain fatty acids (VLCFAs) exhibiting $>22$ carbons are too long to be metabolized in the mitochondria, and must be metabolized in peroxisomes [38]. Activated PPAR $\alpha$ is known to promote peroxisomes in mice, and increase levels of fatty acid oxidation [38-40]. We noticed that peroxisome is ranked $8^{\text {th }}$ in the analysis of enrichment pathways (Fig. 3b). Many enzymes related to fatty acid $\beta$ oxidation that are located in peroxisomes, including Acox, Acaa1, Ehhadh, Crat, Crot, are known to be upregulated (Supplementary Table 2). Another important upregulated protein is Pex11, a protein that regulates peroxisome division to increase peroxisome abundance [41, 42].

Catalase is one of the most important enzymes involved in protecting the cell from oxidative damage by catalyzing the decomposition of hydrogen peroxide. Due to localization in peroxisomes, it is usually used as a marker for peroxisomes $[43,44]$. We detected catalase in the liver by immunohistochemistry (IHC) and Western immunoblotting to confirm peroxisome 
1 abundance. Results obtained for catalase by IHC and immunoblotting suggest a higher 2 abundance of peroxisomes after curcumin supplementation (Figs. 5a-b). The overexpression of 3 catalase also reflects an increase in fatty acid metabolic activity and oxidative stress in 4 hepatocytes.

\section{Figure 5}

a

Catalase
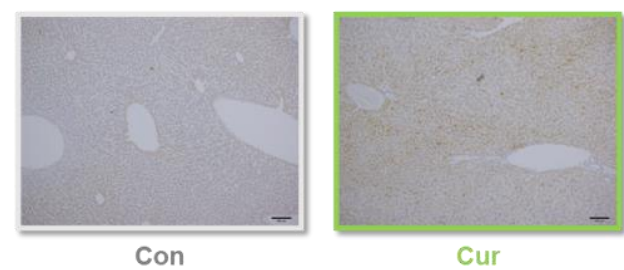

Cur

b

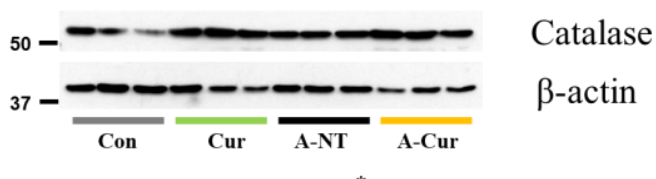

\section{d}

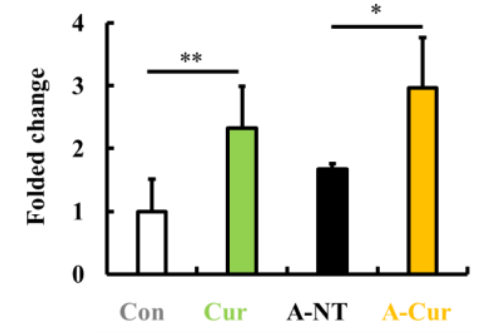

Proteomics analysis of the $75 \mathrm{kD}$ band

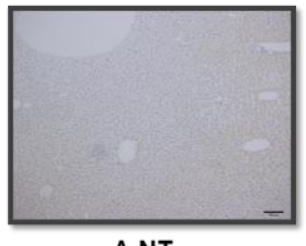

A-NT

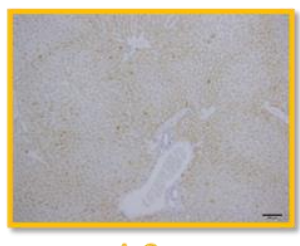

A-Cur

C

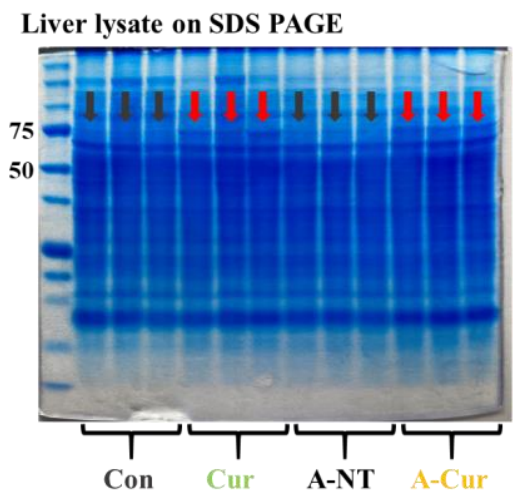

\begin{tabular}{|c|c|c|c|c|c|c|}
\hline$\#$ & Identified Proteins (369) & Gene Name & Con & Cur & A-NT & A-Cur \\
\hline 1 & Peroxisomal bifunctional enzyme & Ehhadh & 123 & 581 & 107 & 577 \\
\hline 2 & Carbamoyl-phosphate synthase & Cpsl & 154 & 139 & 225 & 169 \\
\hline 3 & Serotransferrin & Tf & 151 & 121 & 163 & 134 \\
\hline$\ldots$ & $\ldots$ & & & & & \\
\hline 8 & Peroxisomal multifunctional enzyme type 2 & Hsdl7b4 & 58 & 81 & 56 & 88 \\
\hline$\ldots$ & $\ldots$ & & & & & \\
\hline 13 & Long-chain-fatty-acid--CoA ligase 1 & Acsll & 39 & 53 & 44 & 64 \\
\hline$\ldots$ & $\ldots$ & & & & & \\
\hline
\end{tabular}

Figure 5. Peroxisome and fatty acid oxidative enzymes are increased in the liver.

IHC (a) and Western blot (b) results confirmed that catalase was significantly increased after curcumin supplementation. (c) The protein band near $75 \mathrm{kD}$ of liver lysates exhibited different expression levels for the commercial diet and curcumin diet groups. (d) Three peroxisomal proteins were identified in the band that were found to be upregulated by LC-MS/MS. Data are mean \pm SD. The Tukey-Kramer method was used for multiple comparisons; $* \mathrm{P}<0.05, * * \mathrm{P}<0.01$.

In addition, we unexpectedly found that the protein band observed at a molecular weight of $75 \mathrm{kD}$ is significantly increased in SDS PAGE of the liver extracts stained by Coomassie brilliant blue (Fig. 5c). We analyzed the proteins in this band by proteomic LC-MS/MS analysis and determined that it was comprised of several peroxisomal proteins related to fatty acid oxidation, including Ehhadh, Hsd17b4 and Acsll (Supplementary Table 3). These results demonstrate that curcumin regulates peroxisome abundance via PPAR $\alpha$ activation. 


\section{Discussion :}

2 In previous studies, curcumin was found to exert physiological activities involved in the 3 regulation of several transcription factors (PPARr, NFKB, AP-1, STAT, etc.) and their signaling 4 pathways $[15,16]$. In the present experiment, we found that the gene expression changes in mouse liver after curcumin supplementation are centered on the PPAR signaling pathway. In the enrichment pathway analysis, most of the DEGs involved in retinol metabolism, metabolic pathways, fatty acid degradation and the peroxisome pathway are also downstream of PPAR $\alpha$. Until now, research on curcumin has mainly focused on the activation of PPARr, and it has been demonstrated that curcumin participates in glucose and lipid metabolism, inflammatory cytokine production, and inhibiting tumor cell proliferation via PPARr [16]. Although some studies have found that PPAR $\alpha$ expression is increased after curcumin intake [45, 46], there is currently a lack of further experimental results revealing any connection between curcumin and PPAR $\alpha$ pathway activation. Our results complement the theoretical system suggesting that curcumin regulates the transcription of many genes in the liver depending centrally on PPAR $\alpha$ activation and broadly affects fatty acid transport and catabolism. These changes in genes and proteins levels may be involved, in which curcumin regulates the occurrence and development of various diseases or phenotypes, such as amyloidosis, changes in HDL and triglycerides, oxidative stress, peroxisome proliferation and hepatocyte hypertrophy, etc. (Fig. 6).

Figure 6

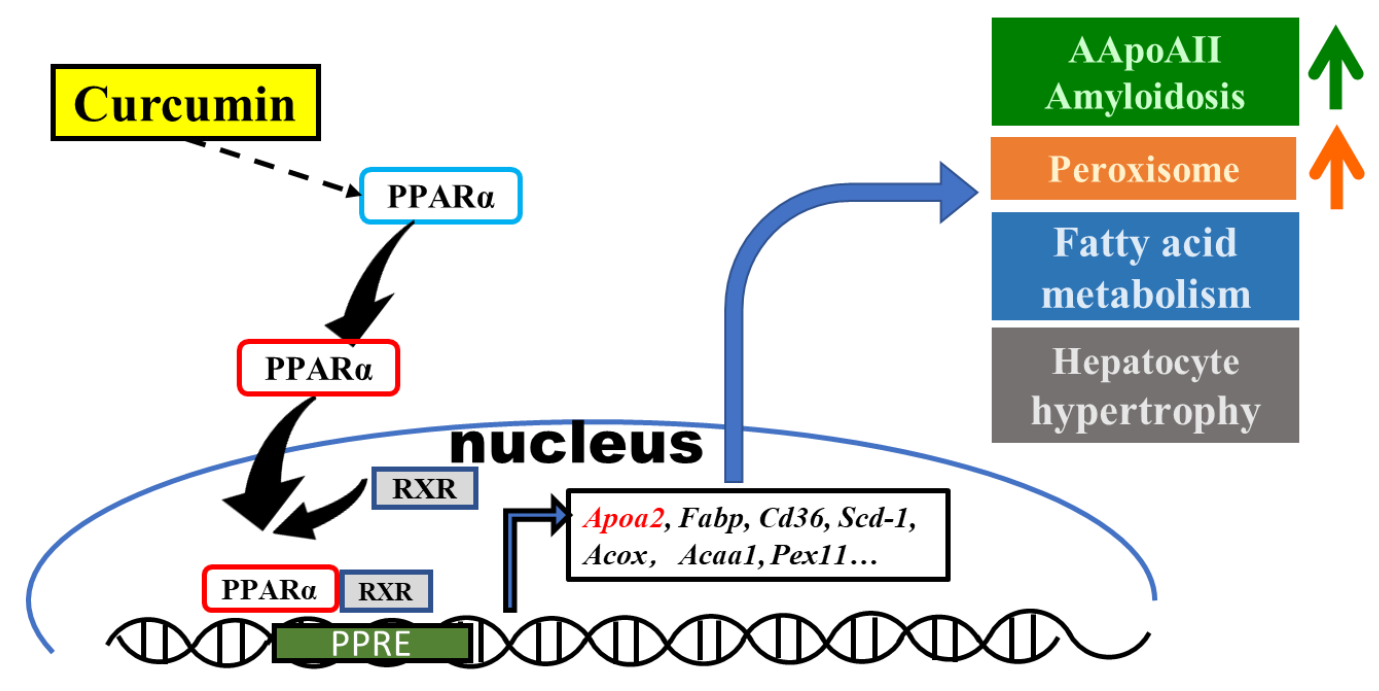

Figure 6. Schematic diagram of the effect of curcumin mediated by PPARa in mouse liver.

Curcumin regulates gene expression via PPARa activation and exhibits biological activities involved in amyloidosis, peroxisome proliferation, lipid metabolism, and hepatocyte hypertrophy. 
Due to the unexpected promotion of AApoAII amyloid deposition by curcumin in this study, we suspect that the anti-amyloid effect of curcumin is not applicable to all types of amyloidosis. To better understand the findings of the present study, we suggest that the effects of curcumin on progression of amyloidosis should be divided into two aspects, one of which is a pro-amyloidosis effect. In this experiment, the plasma concentration of ApoA-II increased almost three-fold (Fig. 2a) via PPAR $\alpha$ pathway activation. Levels of amyloid protein are the most important factor for progression of amyloidosis, and suppression of amyloid protein levels is the main target for treatment of various systemic amyloidosis, including AA, AL, ATTR and dialysis-related $\mathrm{A} \beta_{2} \mathrm{M}$ amyloidosis [2, 3, 27, 47, 48]. In ApoA-II transgenic mice, the serum concentration of ApoA-II increased two-fold and AApoAII amyloid deposition was notably accelerated [30]. However, calorie restriction (diet) decreased the ApoA-II/ApoA-I ratio in serum and suppressed amyloidosis [49]. Thus, we believe that the pro-amyloid effect of curcumin observed here is mainly caused by increased ApoA-II levels.

The other aspect is the anti-amyloid effect of curcumin. Curcumin has shown general affinity for the amyloid protein structure and it has been demonstrated that this binding is efficient to maintain stability of the amyloid protein and inhibit the formation of insoluble amyloid fibrils [4, $9,10]$. In addition to direct binding to amyloid proteins, it has also been shown that curcumin activates autophagy and macrophages in some studies to reduce the dysfunction caused by amyloid proteins $[50,51]$. Moreover, it was reported that some molecules elevate lysosome biosynthesis via activation of PPAR $\alpha$ and accelerate the clearance of amyloid proteins and protein aggregates $[52,53]$. Compared with previous data, we noticed that amyloid deposition in Apoa $^{c}$ transgenic mice with twice the serum concentration of ApoA-II is more severe than in those with greater ApoA-II concentrations in this study [30]. In transgenic mice injected with 1 $\mu \mathrm{g}$ AApoAII fibrils for 8 and 12 weeks, the average amyloid score in the liver and spleen at 12 weeks and the amyloid index at 8 weeks was 3.4, 4 and 3, respectively, compared with values of 2, 3.4 and 1.5, respectively, in curcumin-supplemented mice. We believe that this difference demonstrates that curcumin may also exert a certain anti-amyloid ability in this experiment, but it is difficult to evaluate and further analyses should be carried out in the future.

Curcumin interacts with various cellular metabolic pathways by activating PPARs. Most biological process, such as metabolic control and defect, involve complex molecular interactions and are regulated via various signaling pathways. When amyloid proteins play functional roles in certain metabolic pathways, progression of amyloidosis may be accelerated or decelerated via treatments that modulate such metabolic pathways. As ApoA-II interacts particularly with lipid metabolism, it is likely that amyloid deposition was increased by metabolic changes caused by curcumin, including acceleration of B-oxidation of fatty acids, reduction of lipogenesis and increased synthesis of apolipoproteins. 
In addition, we think the peroxisome proliferation is the most important factor that explains the hepatocyte and liver hypertrophy. There was no significant change in food intake between the four groups investigated in our experiments (Supplementary Fig. 9), and no significant lipid deposition was observed in the liver (Supplementary Fig. 3). Peroxisomes in liver parenchymal cells are very few, and represent $<2 \%$ of cytoplasmic volume under physiological conditions and contribute about $35 \%$ of the $\mathrm{H}_{2} \mathrm{O}_{2}$-production levels. However, peroxisomes in the liver in the presence of peroxisome agonists may occupy as much as $25 \%$ of the cytoplasmic volume [54]. In previous studies, long-term administration of PPAR $\alpha$ agonists was shown to induce disordered peroxisome proliferation, liver hypertrophy and liver tumors in mice [55]. In our experiments, overexpression of catalase and peroxisomal proteins suggests that curcumin promotes peroxisome proliferation mediated by PPAR $\alpha$. The hypertrophic changes in the hepatocyte nucleus we observed in histological sections are consistent with the early pathological changes of hepatocyte heterogeneity.

Peroxisomes are a conserved organelle and play a key role in lipid metabolism and redox homeostasis in both plants and mammals [54, 56, 57]. According to the proliferation mode of peroxisomes, the process of peroxisome fission is a three-step process involving peroxisome elongation, constriction, and scission. Pex11 is essential for this process and its overexpression causes peroxisome proliferation, while its deletion causes a decrease in the number of peroxisomes [58]. Overexpression of Pex11 in curcumin-supplemented mice suggests that Pex11 may play a key role in peroxisome proliferation mediated by PPAR $\alpha$ and curcumin. Moreover, proteins such as Acsl1, Acaal, and Ehhadh upregulated by curcumin in this experiment are localized in the peroxisome matrix and may be involved in this process.

A hallmark of eukaryotic cells is the presence of membrane-bound organelles, such as endoplasmic reticulum (ER), mitochondria, and peroxisomes. Such distinct compartments create special micro-environments for more efficient metabolic reactions. To coordinate complex metabolic processes and signal transduction, there are functional interplays between various organelles. Due to the central metabolic role, it was shown that peroxisomes interact with many organelles involved in cellular lipid metabolism, such as the ER, mitochondria, lysosomes and lipid droplets [59-61]. There is also functional interplay between peroxisomes and the nucleus, which may also involve signaling via $\mathrm{H}_{2} \mathrm{O}_{2}[62,63]$.

Other means by which peroxisome proliferation affects ER or mitochondrial functions are also known. However, the mechanism by which curcumin activates PPARs is not yet clear. There are two means of activating PPARs, namely ligand-dependent and ligand-independent. In the ligand-dependent manner, the molecular shape of PPARs is modified by ligand binding in the cytoplasm, and PPARs enter into the nucleus. In a ligand-independent manner, PPARs can be phosphorylated by protein kinases to induce a structural change of phosphorylated PPARs, even 
1 in the absence of ligands [20-22]. Further experiments are needed to confirm whether curcumin

2 directly binds and activates PPARs as an exogenous ligand, or whether activation involves a

3 ligand-independent pathway.

$4 \quad$ PPAR $\alpha$ and PPARr involve different aspects of metabolic pathways, such as decomposition or storage of fatty acids, fatty acid-based energy production or glucose-based energy production. PPAR $\alpha$ agonists (e.g., fibrates) or PPARr agonists (e.g., thiazolidinedione) play important roles in the treatment of hyperlipidemia and type 2 diabetes in the clinic. PPAR $\alpha / \mathrm{r}$ dual agonists are also under development to treat more complex metabolic diseases, but some exhibit side effects and cause liver or cardiac dysfunction $[64,65]$. In clinical trials, it has been demonstrated that supplementation with curcumin at a high dose is safe in humans [66, 67]. Improving the molecular structure of drugs based on that of curcumin offers the possibility to produce dual or specific agonists without side effects.

13 Taken together, our results demonstrate the novel agonistic effect of curcumin on PPAR $\alpha$. We identified specific effects of curcumin on mice, including promotion of AApoAII amyloidosis and peroxisome proliferation. Curcumin is involved in various physiological activities mediated by PPAR activation, leading to regulation of genes participating in the PPAR pathway. The beneficial use of curcumin based on these particular abilities requires further consideration. The development of derivative agents based on curcumin with high bioavailability or specific effects may have far-reaching significance for the treatment of diseases such as amyloidosis, hyperlipidemia, type 2 diabetes and other metabolic disorders.

\section{Materials and Methods:}

\section{Animals \& Drug Administration}

R1.P1-Apoa $2^{c}$ congenic mice were used in this study, which carry the amyloidogenic type c allele $\left(\right.$ Apoa $\left.^{c}\right)$ of amyloidosis-susceptible SAMP1 strain on a genetic background of the SAMR1 strain. R1.P1-Apoa ${ }^{c}$ mice exhibit a normal aging process and develop accelerated AApoAII amyloidosis by oral or intravenous administration of AApoAII fibrils [24]. Mice were maintained under SPF conditions at $24 \pm 2{ }^{\circ} \mathrm{C}$ with a light-controlled regimen (12-hour light/dark cycle) in the Division of Animal Research, Research Center for Supports to Advanced Science, Shinshu University. The mice were fed a commercial diet (Con group and A-NT group) or curcumin diet (Cur group and A-Cur group) and tap water ad libitum. The commercial diet is a MF diet (Oriental Yeast, Tokyo, Japan) and the curcumin diet is the MF diet supplemented with $0.5 \%$ or $2 \% \mathrm{w} / \mathrm{w}$ curcumin (Wako, Osaka, Japan). Three to 5 R1.P1-Apoa $2^{c}$ congenic mice were housed in a single cage. Female mice were used for experiments to avoid the anticipated adverse impacts due to fighting among male mice. Mice were sacrificed by cardiac puncture under deep sevoflurane anesthesia after 8 weeks and 12 
1 weeks of curcumin intake. Plasma and half of the major organs (heart, liver, spleen, stomach, small intestine, tongue, skin, lung and kidney) were snap-frozen by liquid nitrogen and stored at $-80^{\circ} \mathrm{C}$ for biochemical analysis. The remaining organs were fixed in $10 \%$ neutral buffered formalin followed by embedding in paraffin for histochemical analysis. All experiments were approved by the Committee for Animal Experiments of Shinshu University (Approval No. 280086).

\section{Induction of AApoAII amyloidosis}

9 AApoAII amyloid fibrils were isolated using Pras' method [68] from the livers of R1.P1-Apoa2 ${ }^{c}$ mice having severe amyloid deposits. Mice in the amyloid-induced groups were injected with $1 \mu \mathrm{g}$ amyloid fibrils into the tail vein for induction of AApoAII amyloidosis at 8 weeks of age. AApoAII fibrils were sonicated before use and the injection was performed immediately.

\section{Evaluation of amyloid deposition}

15 Amyloid deposits were detected in paraffin organ sections stained with a saturated solution of $1 \%$ 16 Congo red dye. An amyloid score (from 0 to 4) in each organ was determined semi-quantitatively as described previously [69] under polarizing light microscopy (LM) (Axioskop 2, Carl Zeiss, Tokyo, Japan). Two observers, with no information of the Congo red stained tissues, graded the degree of amyloid deposition in each mouse, separately. The degree of amyloid deposition in each mouse was represented by an amyloid index (AI), which is the average of the amyloid scores in seven organs (heart, liver, spleen, stomach, small intestine, tongue, and skin).

\section{Hepatocyte size in each group}

To analyze the hepatocyte size in each mouse quantitatively, we captured 5 images of each section selected randomly at $400 \times$ magnification and determined the average hepatocyte size in each image by calculating the total area divided by the cell counts using an image processing program (NIH ImageJ software, version 1.61). We determined the average hepatocyte size of each mouse and then performed a statistical analysis between all four groups.

\section{Immunohistochemistry and immunofluorescence analysis}

We detected AApoAII deposition and catalase by immunohistochemistry (IHC) following a previously described method [49]. Antiserum against mouse ApoA-II was produced against guanidine hydrochloride-denatured AApoAII in our laboratory [70] and applied at a dilution ratio of 1:3000. Catalase antibody was applied (1:500, GTX110704, GeneTex Inc., CA, USA) to reveal the degree of peroxisome change in the liver. After incubation overnight at $4^{\circ} \mathrm{C}$ with the 
1 primary antibody, the sections were incubated with the biotinylated secondary antibody (1:300,

2 DAKO, Glostrup, Denmark) for $1 \mathrm{~h}$ at room temperature. Target proteins were identified by the

3 horseradish peroxidase-labeled streptavidin-biotin method (1:300, DAKO). In the

4 immunofluorescence experiments, the sections were incubated with the PPAR $\alpha$ antibody (1:500,

5 GTX101098, GeneTex Inc., CA, USA) overnight and incubated with Alexa Fluor ${ }^{\mathrm{TM}} 488$ goat

6 anti-rabbit antibody (1:500, Thermo Fisher Scientific, Japan) for $1 \mathrm{~h}$ at room temperature and

7 incubated with DAPI for $10 \mathrm{~min}$. Images were captured immediately using a confocal laser

8 fluorescence microscope (LSM 880 with Airyscan, Carl Zeiss, Germany). In a negative control

9 section, the primary antibody was omitted to confirm the specificity of staining.

\section{Lipid metabolism analysis}

12 Lipid metabolism levels were determined using quantitative assay kits by means of HDL cholesterol, total cholesterol and triglycerides concentrations in the plasma with the instructions provided by the manufacturer (HDL-cholesterol E test, 431-52501; Total-cholesterol E test, 439-17501; TG E test, 432-40201, FUJI FILM Wako, Osaka, Japan).

\section{AST and ALT detection}

18 Two hundred microliters $(200 \mu \mathrm{L})$ of frozen mouse plasma per mouse was sent to Nagahama life science laboratory (Oriental Yeast, Tokyo, Japan) for determination of AST and ALT levels. The laboratory provided a test report.

\section{Immunoblot analysis}

We measured proteins levels by western blotting as described previously [30, 49]. To determine plasma levels of ApoA-II, ApoA-I, ApoE, $0.5 \mu \mathrm{L}$ samples from each mouse were separated by Tris-Tricine/SDS-16.5\% or $15 \%$ polyacrylamide gel electrophoresis (PAGE). After electrophoresis, proteins were transferred to a polyvinylidene difluoride (PVDF) membrane (Immobilon, $0.2 \mu \mathrm{m}$ pore, Millipore Corp., MA, USA) and incubated overnight at $4{ }^{\circ} \mathrm{C}$ with primary antibody solution containing polyclonal rabbit anti-mouse ApoA-II antiserum (diluted 1:3000) or the ApoA-I antiserum (diluted 1:4000) produced in our laboratory, or ApoE antibody (1:500, Santa Cruz, San Francisco, CA, USA). Next, horseradish peroxidase-conjugated anti-rabbit IgG (Code \#7074, Cell Signaling Technology Inc., Danvers MA, USA) (1:3000) was used for 1-h incubation at room temperature and target proteins were detected by the enhanced chemiluminescence (ECL) method. Thirty micrograms $(30 \mu \mathrm{g})$ of liver lysates were separated on Tris-Tricine/SDS-12\% PAGE to determine levels of PPARa (1:3000, GTX101098, GeneTex Inc.), $\beta$-actin (1:3000, GTX110564, GeneTex Inc.) and catalase (1:3000, GTX110704, GeneTex Inc., CA, USA). Target protein levels were analyzed using the NIH ImageJ software. 


\section{RNA sequence analysis}

3 We selected liver samples from the 12-week group for RNA sequence analysis, which exhibit more obvious amyloid deposition in amyloid-induced animals and no abnormal changes in AST and ALT levels. Ten milligrams $(10 \mathrm{mg})$ of each mouse liver stored at $-80^{\circ} \mathrm{C}$ was homogenized in TRIzol RNA isolation reagent (Invitogen-Thermo Fisher, Tokyo Japan) and pooled into 4 sample tubes (Con, Cur, A-NT and A-Cur groups, $\mathrm{N}=3-5$ ), and the samples were sent to Filgen (Nagoya, Japan). Total RNA was extracted and the RNA purity and integrity were confirmed using a Bioanalyzer 2100 system (Agilent Technology, Santa Clara, CA USA). mRNA sequencing analysis was performed with an Illumina next generation sequencing platform. Sequencing count data were analyzed using the DESeq2 software to determine the significant DEGs among the different groups. Biological functions into which DEGs accumulated were analyzed using an annotation database (Gene Ontology and KEGG Pathway Database) to elucidate the mechanism of the effects of curcumin supplementation.

\section{Gene expression analysis}

17 We followed a previously described method to confirm mRNA expression levels [71]. 18 Quantitative real-time qPCR analysis was carried out using an ABI PRISM 7500 Sequence 19 Detection system (Applied Biosystems, New York, USA) with SYBR Green (TaKaRa Bio, Tokyo, Japan). The $\beta$-actin gene was used to normalize gene expression. The forward and reverse primer sequences for real-time PCR are listed in Supplementary Table 4. Chemical reagents used in the experiments, unless otherwise specified, were obtained from Wako Pure Chemical Industries Ltd. (Osaka, Japan).

\section{Nano-flow liquid chromatography-ion trap mass spectrometry (LC-MS/MS)}

Thirty micrograms $(30 \mu \mathrm{g})$ of liver lysates were separated on Tris-Tricine/SDS-12\% PAGE and stained with Coomassie brilliant blue for $20 \mathrm{~min}$. The stained bands near $75 \mathrm{kD}$ were excised and soaked in $50 \mathrm{mM}$ Tris- $\mathrm{HCl}, \mathrm{pH} 8.0$, containing $50 \%$ acetonitrile for $30 \mathrm{~min}$. The gel was dried in a Speed-Vac (Savant) and incubated in $50 \mathrm{mM}$ triethylammonium bicarbonate containing proteomics grade trypsin (Sigma-Aldrich, Tokyo, Japan) at $37^{\circ} \mathrm{C}$ for $20 \mathrm{~h}$. The digests were extracted from the gel with $100-200 \mu 1$ of $0.1 \%$ TFA containing $60 \%$ acetonitrile. These extracts were evaporated in a Speed-Vac and stored at $-80^{\circ} \mathrm{C}$ until assayed. EASY-nLC 1200 (Thermo Fisher Scientific Inc., Waltham, MA, USA). A packed nano-capillary column, NTCC-360/75-3-123 (0.075 mm I.D. $\times 125 \mathrm{~mm} \mathrm{~L}$, particle diameter $3 \mu \mathrm{m}$, Nikkyo 
1 gradient of acetonitrile for $80 \mathrm{~min}$. Eluted peptides were directly detected with an ion trap mass spectrometer (QExactive HF; Thermo Fisher Scientific Inc., Waltham, MA, USA). For ionization, a spray voltage of $2.0 \mathrm{kV}$ and capillary temperature of $250^{\circ} \mathrm{C}$ was used. The mass acquisition method consisted of one full MS survey scan with an Orbitrap resolution of 60,000, followed by an MS/MS scan of the most abundant precursor ions from the survey scan with an Orbitrap resolution of 15,000. Dynamic exclusion for the MS/MS was set to $30 \mathrm{sec}$. An MS scan range of $350-1800 \mathrm{~m} / \mathrm{z}$ was employed in the positive ion mode, followed by data-dependent MS/MS using the HCD operating mode on the top 15 ions in order of abundance. The data were analyzed with Proteome Discoverer (Thermo Fisher Scientific Inc., Waltham, MA, USA), Mascot software (Matrix Science Inc., Boston, MA, USA) and Scaffold software (Proteome Software, Inc., Oregon, USA). Swissprot and GenBank databases were used.

\section{Statistical analyses}

For comparison of parametrical data, one-way analysis of variance (ANOVA) with Tukey's test was performed using the SPSS 26.0 software package (Abacus Concepts, Berkley, CA USA). For comparison of nonparametric data, the Kruskal-Wallis test with the Steel-Dwass test was performed. $\mathrm{P}$ values $<0.05$ were considered to be statistically significant.

\section{Data Availability}

The data used to support the findings of this study are included within the article. All other data supporting the findings of this study will be made available upon reasonable request to the corresponding authors.

\section{Conflicts of Interest}

The authors declare that they have no competing interests.

\section{Authors' Contributions}

J.D. and K.H. conceived and designed the experiments. J.D., Y.L., F.K., X.C., Y.I and J.H. performed the experiments and were responsible for data acquisition and analysis. J.D., H.M., and K.H. analyzed the data. M.M. interpreted the data and experimental methods. J.D. and K.H. wrote the manuscript. All authors reviewed the manuscript and approved the manuscript for publication.

\section{Acknowledgments}

This work was supported in part by Grants-in-Aid for Scientific Research (B) 17H04063 and 
1 Science and Technology, Japan, The authors thank Drs. Kiyoshi Matsumoto and Takahiro

2 Yoshizawa, Hiroto Yamanaka, and Ms. Kayo Suzuki (Research Center for Supports to

3 Advanced Science, Shinshu University) for animal care and technical assistance in the 4 preparation of tissue sections.

5

6

\section{References}

1. Benson MD, Buxbaum JN, Eisenberg DS, Merlini G, Saraiva MJM, Sekijima Y, Sipe JD, Westermark P: Amyloid nomenclature 2018: recommendations by the International Society of Amyloidosis (ISA) nomenclature committee. Amyloid 2018, 25(4):215-219. DOI: 10.1080/13506129.2018.1549825

2. Merlini G, Bellotti V: Molecular Mechanisms of Amyloidosis. New England Journal of Medicine 2003, 349(6):583-596. DOI: 10.1056/NEJMra023144

3. Knowles TPJ, Vendruscolo M, Dobson CM: The amyloid state and its association with protein misfolding diseases. Nature Reviews Molecular Cell Biology 2014, 15(6):384-396. DOI: $10.1038 / \mathrm{nrm} 3810$

4. Stefani M, Rigacci S: Protein folding and aggregation into amyloid: the interference by natural phenolic compounds. In: Int J Mol Sci. vol. 14; 2013: 12411-12457. DOI: 10.3390/ijms140612411

5. Lim GP, Chu T, Yang F, Beech W, Frautschy SA, Cole GM: The Curry Spice Curcumin Reduces Oxidative Damage and Amyloid Pathology in an Alzheimer Transgenic Mouse. The Journal of Neuroscience 2001, 21(21):8370. DOI: 10.1523/JNEUROSCI.21-21-08370.2001

6. Ferreira N, Santos SA, Domingues MR, Saraiva MJ, Almeida MR: Dietary curcumin counteracts extracellular transthyretin deposition: insights on the mechanism of amyloid inhibition. Biochimica et biophysica acta 2013, 1832(1):39-45. DOI: 10.1016/j.bbadis.2012.10.007

7. Ferreira N, Gonçalves NP, Saraiva MJ, Almeida MR: Curcumin: A multi-target disease-modifying agent for late-stage transthyretin amyloidosis. Scientific Reports 2016, 6(1):26623. DOI: 10.1038/srep26623

8. Ma Q-L, Zuo X, Yang F, Ubeda OJ, Gant DJ, Alaverdyan M, Teng E, Hu S, Chen P-P, Maiti $\mathrm{P}$ et al: Curcumin Suppresses Soluble Tau Dimers and Corrects Molecular Chaperone, Synaptic, and Behavioral Deficits in Aged Human Tau Transgenic Mice. Journal of Biological Chemistry 2013, 288(6):4056-4065. DOI: 10.1074/jbc.M112.393751

9. Ahmad B, Borana MS, Chaudhary AP: Understanding curcumin-induced 
modulation of protein aggregation. International journal of biological macromolecules 2017, 100:89-96. DOI: 10.1016/j.ijbiomac.2016.06.053

10. Hafner-Bratkovič I, Gašperšič J, Šmid LM, Bresjanac M, Jerala R: Curcumin binds to the $\alpha$-helical intermediate and to the amyloid form of prion protein - a new mechanism for the inhibition of PrPSc accumulation. Journal of Neurochemistry 2008, 104(6):1553-1564. DOI: 10.1111/j.1471-4159.2007.05105.X

11. Song X-J, Zhou H-Y, Sun Y-X, Huang H-C: Inhibitory effects of curcumin on H2O2-induced cell damage and APP expression and processing in SH-SY5Y cells transfected with APP gene with Swedish mutation. Molecular Biology Reports 2020, 47(3):2047-2059. DOI: 10.1007/s11033-020-05305-w

12. Zheng K, Dai X, Xiao N, Wu X, Wei Z, Fang W, Zhu Y, Zhang J, Chen X: Curcumin Ameliorates Memory Decline via Inhibiting BACE1 Expression and $\beta$-Amyloid Pathology in 5×FAD Transgenic Mice. Molecular neurobiology 2017, 54(3):1967-1977. DOI: 10.1007/s12035-016-9802-9

13. Golombick T, Diamond TH, Manoharan A, Ramakrishna R: Stabilisation of Laryngeal AL Amyloidosis with Long Term Curcumin Therapy. Case Reports in Hematology 2015, 2015:910528. DOI: 10.1155/2015/910528

14. Small GW, Siddarth P, Li Z, Miller KJ, Ercoli L, Emerson ND, Martinez J, Wong KP, Liu J, Merrill DA et al: Memory and Brain Amyloid and Tau Effects of a Bioavailable Form of Curcumin in Non-Demented Adults: A Double-Blind, Placebo-Controlled 18-Month Trial. The American journal of geriatric psychiatry : official journal of the American Association for Geriatric Psychiatry 2018, 26(3):266-277. DOI: 10.1016/j.jagp.2017.10.010

15. Liczbiński P, Michałowicz J, Bukowska B: Molecular mechanism of curcumin action in signaling pathways: Review of the latest research. Phytother Res 2020, 34(8):1992-2005. DOI: 10.1002/ptr.6663

16. Shishodia S: Molecular mechanisms of curcumin action: Gene expression. BioFactors 2013, 39(1):37-55. DOI: 10.1002/biof.1041

17. $\mathrm{Xu} \mathrm{J}, \mathrm{Fu} \mathrm{Y}, \mathrm{Chen} \mathrm{A}$ : Activation of peroxisome proliferator-activated receptor- $\gamma$ contributes to the inhibitory effects of curcumin on rat hepatic stellate cell growth. American Journal of Physiology-Gastrointestinal and Liver Physiology 2003, 285(1):G20-G30. DOI: 10.1152/ajpgi.00474.2002

18. Pyper SR, Viswakarma N, Yu S, Reddy JK: PPARa: Energy Combustion, Hypolipidemia, Inflammation and Cancer. Nuclear Receptor Signaling 2010, 8(1):nrs.08002. DOI: 10.1621/nrs.08002

19. Monsalve FA, Pyarasani RD, Delgado-Lopez F, Moore-Carrasco R: Peroxisome 
Proliferator-Activated Receptor Targets for the Treatment of Metabolic Diseases. Mediators of Inflammation 2013, 2013:549627. DOI: 10.1155/2013/549627

20. Lamichane S, Dahal Lamichane B, Kwon S-M: Pivotal Roles of Peroxisome Proliferator-Activated Receptors (PPARs) and Their Signal Cascade for Cellular and Whole-Body Energy Homeostasis. Int $J$ Mol Sci 2018, 19(4):949. DOI: 10.3390/ijms 19040949

21. Harmon GS, Lam MT, Glass CK: PPARs and Lipid Ligands in Inflammation and Metabolism. Chemical Reviews 2011, 111(10):6321-6340. DOI: 10.1021/cr2001355

22. Magadum A, Engel FB: PPARß/ס: Linking Metabolism to Regeneration. Int $J$ Mol Sci 2018, 19(7):2013. DOI: 10.3390/ijms19072013

23. Dai J, Ding X, Miyahara H, Xu Z, Cui X, Igarashi Y, Sawashita J, Mori M, Higuchi K: Suppression of Mouse AApoAII Amyloidosis Progression by Daily Supplementation with Oxidative Stress Inhibitors. Oxidative Medicine and Cellular Longevity 2019, 2019:1263274. DOI: 10.1155/2019/1263274

24. Higuchi K, Naiki H, Kitagawa K, Kitado H, Kogishi K, Matsushita T, Takeda T: Apolipoprotein A-II gene and development of amyloidosis and senescence in a congenic strain of mice carrying amyloidogenic ApoA-II. Laboratory investigation; a journal of technical methods and pathology 1995, 72(1):75-82.

25. Um MY, Hwang KH, Ahn J, Ha TY: Curcumin Attenuates Diet-Induced Hepatic Steatosis by Activating AMP-Activated Protein Kinase. Basic \& Clinical Pharmacology \& Toxicology 2013, 113(3):152-157. DOI: 10.1111/bcpt.12076

26. Maithilikarpagaselvi N, Sridhar MG, Swaminathan RP, Sripradha R, Badhe B: Curcumin inhibits hyperlipidemia and hepatic fat accumulation in high-fructose-fed male Wistar rats. Pharmaceutical Biology 2016, 54(12):2857-2863. DOI: $10.1080 / 13880209.2016 .1187179$

27. Calhoun ME, Burgermeister P, Phinney AL, Stalder M, Tolnay M, Wiederhold K-H, Abramowski D, Sturchler-Pierrat C, Sommer B, Staufenbiel M et al: Neuronal overexpression of mutant amyloid precursor protein results in prominent deposition of cerebrovascular amyloid. Proceedings of the National Academy of Sciences 1999, 96(24):14088-14093. DOI: 10.1073/pnas.96.24.14088

28. Yang Y-S, Su Y-F, Yang H-W, Lee Y-H, Chou JI, Ueng K-C: Lipid-Lowering Effects of Curcumin in Patients with Metabolic Syndrome: A Randomized, Double-Blind, Placebo-Controlled Trial. Phytotherapy Research 2014, 28(12):1770-1777. DOI: 10.1002/ptr.5197

29. Panahi Y, Ahmadi Y, Teymouri M, Johnston TP, Sahebkar A: Curcumin as a potential candidate for treating hyperlipidemia: A review of cellular and metabolic 
mechanisms. Journal of Cellular Physiology 2018, 233(1):141-152. DOI: $10.1002 / \mathrm{jcp} .25756$

30. Ge F, Yao J, Fu X, Guo Z, Yan J, Zhang B, Zhang H, Tomozawa H, Miyazaki J, Sawashita $\mathrm{J}$ et al: Amyloidosis in transgenic mice expressing murine amyloidogenic apolipoprotein A-II (Apoa2c). Laboratory Investigation 2007, 87(7):633-643. DOI: 10.1038/labinvest.3700559

31. Miyahara H, Sawashita J, Ishikawa E, Yang M, Ding X, Liu Y, Hachiya N, Kametani F, Yazaki M, Mori $\mathrm{M}$ et al: Comprehensive proteomic profiles of mouse AApoAII amyloid fibrils provide insights into the involvement of lipoproteins in the pathology of amyloidosis. Journal of Proteomics 2018, 172:111-121. DOI: 10.1016/j.jprot.2017.10.003

32. van der Hoogt CC, de Haan W, Westerterp M, Hoekstra M, Dallinga-Thie GM, Romijn JA, Princen HMG, Jukema JW, Havekes LM, Rensen PCN: Fenofibrate increases HDL-cholesterol by reducing cholesteryl ester transfer protein expression. Journal of Lipid Research 2007, 48(8):1763-1771. DOI: 10.1194/j1r.M700108-JLR200

33. Staels B, Dallongeville J, Auwerx J, Schoonjans K, Leitersdorf E, Fruchart J-C: Mechanism of Action of Fibrates on Lipid and Lipoprotein Metabolism. Circulation 1998, 98(19):2088-2093. DOI: 10.1161/01.cir.98.19.2088

34. Janani C, Ranjitha Kumari BD: PPAR gamma gene - A review. Diabetes \& Metabolic Syndrome: Clinical Research \& Reviews 2015, 9(1):46-50. DOI: 10.1016/j.dsx.2014.09.015

35. Youssef $\mathrm{J}$, Badr $\mathrm{M}$ : Peroxisome proliferator-activated receptors and cancer: challenges and opportunities. British Journal of Pharmacology 2011, 164(1):68-82. DOI: 10.1111/j.1476-5381.2011.01383.x

36. Liu C, Guo Q, Lu M, Li Y: An experimental study on amelioration of dyslipidemia-induced atherosclesis by Clematichinenoside through regulating Peroxisome proliferator-activated receptor- $\alpha$ mediated apolipoprotein A-I, A-II and C-III. European Journal of Pharmacology 2015, 761:362-374. DOI: 10.1016/j.ejphar.2015.04.015

37. Shah A, Rader DJ, Millar JS: The effect of PPAR-a agonism on apolipoprotein metabolism in humans. Atherosclerosis 2010, 210(1):35-40. DOI: 10.1016/j.atherosclerosis.2009.11.010

38. Islinger M, Voelkl A, Fahimi HD, Schrader M: The peroxisome: an update on mysteries 2.0. Histochemistry and Cell Biology 2018, 150(5):443-471. DOI: 10.1007/s00418-018-1722-5

39. Schrader M, Costello JL, Godinho LF, Azadi AS, Islinger M: Proliferation and fission 
of peroxisomes - An update. Biochimica et Biophysica Acta (BBA) - Molecular Cell Research 2016, 1863(5):971-983. DOI: 10.1016/j.bbamcr.2015.09.024

40. Lee SS, Pineau T, Drago J, Lee EJ, Owens JW, Kroetz DL, Fernandez-Salguero PM, Westphal H, Gonzalez FJ: Targeted disruption of the alpha isoform of the peroxisome proliferator-activated receptor gene in mice results in abolishment of the pleiotropic effects of peroxisome proliferators. Molecular and Cellular Biology 1995, 15(6):3012-3022. DOI: 10.1128/mcb.15.6.3012

41. Weng H, Ji X, Naito Y, Endo K, Ma X, Takahashi R, Shen C, Hirokawa G, Fukushima Y, Iwai N: Pex11a deficiency impairs peroxisome elongation and division and contributes to nonalcoholic fatty liver in mice. American Journal of Physiology-Endocrinology and Metabolism 2013, 304(2):E187-E196. DOI: 10.1152/ajpendo.00425.2012

42. Knoblach B, Rachubinski RA: Phosphorylation-dependent Activation of Peroxisome Proliferator Protein PEX11 Controls Peroxisome Abundance. Journal of Biological Chemistry 2010, 285(9):6670-6680. DOI: 10.1074/jbc.M109.094805

43. Goldman BM, Blobel G: Biogenesis of peroxisomes: intracellular site of synthesis of catalase and uricase. Proceedings of the National Academy of Sciences 1978, 75(10):5066-5070. DOI: 10.1073/pnas.75.10.5066

44. Fahimi HD: Cytochemical localization of peroxidatic activity of catalase in rat hepatic microbodies (peroxisomes). Journal of Cell Biology 1969, 43(2):275-288. DOI: $10.1083 / \mathrm{jcb} .43 .2 .275$

45. Kong D, Zhang Z, Chen L, Huang W, Zhang F, Wang L, Wang Y, Cao P, Zheng S: Curcumin blunts epithelial-mesenchymal transition of hepatocytes to alleviate hepatic fibrosis through regulating oxidative stress and autophagy. Redox Biology 2020, 36:101600. DOI: 10.1016/j.redox.2020.101600

46. Zeng K, Tian L, Patel R, Shao W, Song Z, Liu L, Manuel J, Ma X, McGilvray I, Cummins CL et al: Diet Polyphenol Curcumin Stimulates Hepatic Fgf21 Production and Restores Its Sensitivity in High-Fat-Diet-Fed Male Mice. Endocrinology 2016, 158(2):277-292. DOI: 10.1210/en.2016-1596

47. Zhang P, Fu X, Sawashita J, Yao J, Zhang B, Qian J, Tomozawa H, Mori M, Ando Y, Naiki $\mathrm{H}$ et al: Mouse model to study human A beta2M amyloidosis: generation of a transgenic mouse with excessive expression of human beta2-microglobulin. Amyloid 2010, 17(2):50-62. DOI: 10.3109/13506129.2010.483116

48. Ueda M, Ando Y, Hakamata Y, Nakamura M, Yamashita T, Obayashi K, Himeno S, Inoue S, Sato Y, Kaneko $\mathrm{T}$ et al: A transgenic rat with the human ATTR V30M: A novel tool for analyses of ATTR metabolisms. Biochemical and Biophysical Research 
Communications 2007, 352(2):299-304. DOI: 10.1016/j.bbrc.2006.11.045

49. Li L, Sawashita J, Ding X, Yang M, Xu Z, Miyahara H, Mori M, Higuchi K: Caloric restriction reduces the systemic progression of mouse AApoAII amyloidosis. PLOS ONE 2017, 12(2):e0172402. DOI: 10.1371/journal.pone.0172402

50. Zhang L, Fiala M, Cashman J, Sayre J, Espinosa A, Mahanian M, Zaghi J, Badmaev V, Graves MC, Bernard G et al: Curcuminoids enhance amyloid-beta uptake by macrophages of Alzheimer's disease patients. Journal of Alzheimer's disease : JAD 2006, 10(1):1-7. DOI: 10.3233/jad-2006-10101

51. Li H, Zhang Y, Cao L, Xiong R, Zhang B, Wu L, Zhao Z, Chen S-D: Curcumin could reduce the monomer of TTR with Tyr114Cys mutation via autophagy in cell model of familial amyloid polyneuropathy. Drug Des Devel Ther 2014, 8:2121-2128. DOI: 10.2147/DDDT.S70866

52. Ghosh A, Jana M, Modi K, Gonzalez FJ, Sims KB, Berry-Kravis E, Pahan K: Activation of peroxisome proliferator-activated receptor $\alpha$ induces lysosomal biogenesis in brain cells: implications for lysosomal storage disorders. Journal of Biological Chemistry 2015, 290(16):10309-10324. DOI: 10.1074/jbc.M114.610659

53. Chandra S, Jana M, Pahan K: Aspirin Induces Lysosomal Biogenesis and Attenuates Amyloid Plaque Pathology in a Mouse Model of Alzheimer's Disease via PPARa. The Journal of Neuroscience 2018, 38(30):6682-6699. DOI: 10.1523/JNEUROSCI.0054-18.2018

54. Yeldandi AV, Rao MS, Reddy JK: Hydrogen peroxide generation in peroxisome proliferator-induced oncogenesis. Mutation Research/Fundamental and Molecular Mechanisms of Mutagenesis 2000, 448(2):159-177. DOI: 10.1016/s0027-5107(99)00234-1

55. Corton JC, Peters JM, Klaunig JE: The PPARa-dependent rodent liver tumor response is not relevant to humans: addressing misconceptions. Archives of Toxicology 2018, 92(1):83-119. DOI: 10.1007/s00204-017-2094-7

56. Farré J-C, Mahalingam SS, Proietto M, Subramani S: Peroxisome biogenesis, membrane contact sites, and quality control. EMBO reports 2019, 20(1):e46864. DOI: $10.15252 / \mathrm{embr} .201846864$

57. Schrader $M$, Kamoshita $M$, Islinger $M$ : Organelle interplay-peroxisome interactions in health and disease. Journal of Inherited Metabolic Disease 2020, 43(1):71-89. DOI: $10.1002 /$ jimd. 12083

58. Erdmann R, Blobel G: Giant peroxisomes in oleic acid-induced Saccharomyces cerevisiae lacking the peroxisomal membrane protein Pmp27p. Journal of Cell Biology 1995, 128(4):509-523. DOI: 10.1083/jcb.128.4.509 
1 59. Islinger M, Godinho L, Costello J, Schrader M: The different facets of organelle interplay-an overview of organelle interactions. Frontiers in Cell and Developmental Biology 2015, 3(56). DOI: 10.3389/fcell.2015.00056

60. Wanders RJA, Waterham HR, Ferdinandusse S: Peroxisomes and Their Central Role in Metabolic Interaction Networks in Humans. Sub-cellular biochemistry 2018, 89:345-365. DOI: 10.1007/978-981-13-2233-4_15

61. Sugiura A, Mattie S, Prudent J, McBride HM: Newly born peroxisomes are a hybrid of mitochondrial and ER-derived pre-peroxisomes. Nature 2017, 542(7640):251-254. DOI: $10.1038 /$ nature21375

62. Schrader M, Grille S, Fahimi HD, Islinger M: Peroxisome interactions and cross-talk with other subcellular compartments in animal cells. Sub-cellular biochemistry 2013, 69:1-22. DOI: 10.1007/978-94-007-6889-5_1

63. Mullineaux PM, Exposito-Rodriguez M, Laissue PP, Smirnoff N: ROS-dependent signalling pathways in plants and algae exposed to high light: Comparisons with other eukaryotes. Free Radical Biology and Medicine 2018, 122:52-64. DOI: 10.1016/j.freeradbiomed.2018.01.033

64. Kim S-H, Hong SH, Park Y-J, Sung J-H, Suh W, Lee KW, Jung K, Lim C, Kim J-H, Kim $\mathrm{H}$ et al: MD001, a Novel Peroxisome Proliferator-activated Receptor $\boldsymbol{\alpha} / \boldsymbol{\gamma}$ Agonist, Improves Glucose and Lipid Metabolism. Scientific Reports 2019, 9(1):1656. DOI: 10.1038/s41598-018-38281-0

65. Kalliora C, Kyriazis ID, Oka S-i, Lieu MJ, Yue Y, Area-Gomez E, Pol CJ, Tian Y,

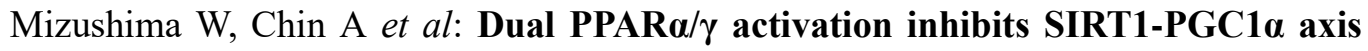
and causes cardiac dysfunction. JCI Insight 2019, 4(17). DOI: 10.1172/jci.insight. 129556

66. Sahebkar A: Curcuminoids for the management of hypertriglyceridaemia. Nature Reviews Cardiology 2014, 11(2):123-123. DOI: 10.1038/nrcardio.2013.140-c1

67. Chen F-Y, Zhou J, Guo N, Ma W-G, Huang X, Wang H, Yuan Z-Y: Curcumin retunes cholesterol transport homeostasis and inflammation response in $\mathrm{M} 1$ macrophage to prevent atherosclerosis. Biochemical and Biophysical Research Communications 2015, 467(4):872-878. DOI: 10.1016/j.bbrc.2015.10.051

68. Pras M, Zucker-Franklin D, Rimon A, Franklin EC: Physical, chemical, and ultrastructural studies of water-soluble human amyloid fibrils. Comparative analyses of nine amyloid preparations. Journal of Experimental Medicine 1969, 130(4):777-795. DOI: $10.1084 /$ jem.130.4.777

69. Xing Y, Nakamura A, Korenaga T, Guo Z, Yao J, Fu X, Matsushita T, Kogishi K, Hosokawa M, Kametani $\mathrm{F}$ et al: Induction of Protein Conformational Change in 
Mouse Senile Amyloidosis. Journal of Biological Chemistry 2002, 277(36):33164-33169. DOI: 10.1074/jbc.M111570200

70. Higuchi K, Matsumura A, Honma A, Takeshita S, Hashimoto K, Hosokawa M, Yasuhira $\mathrm{K}$, Takeda T: Systemic senile amyloid in senescence-accelerated mice. A unique fibril protein demonstrated in tissues from various organs by the unlabeled immunoperoxidase method. Laboratory investigation; a journal of technical methods and pathology 1983, 48(2):231-240.

71. Geng T, Jinko S, Hiroshi K, Shinya Ni, Shigenari H, Nobuyoshi S, Hidekane Y, Mineko Ts, Yaoyong W, Yingye L, Hongming L, Zhe X, Masayuki M, Mitsuaki K, Kazunori H, Toshio T, Shinichi U, Keiichi K: Ubiquinol-10 Supplementation Activates Mitochondria Functions to Decelerate Senescence in Senescence-Accelerated Mice. Antioxidants \& Redox Signaling 2014, 20(16):2606-2620. DOI: 10.1089/ars.2013.5406 


\section{Supplementary Data}

2

\section{Supplementary Figure 1}

\section{Non-amyloid induced groups}

\section{Distilled water}

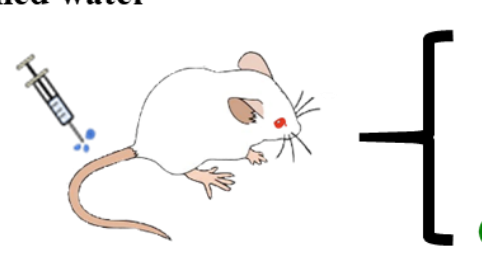

R1.P1-Apoa $2^{c}$ congenic mice (female, 8-week-old)
Con

Cur

(Curcumin diet)
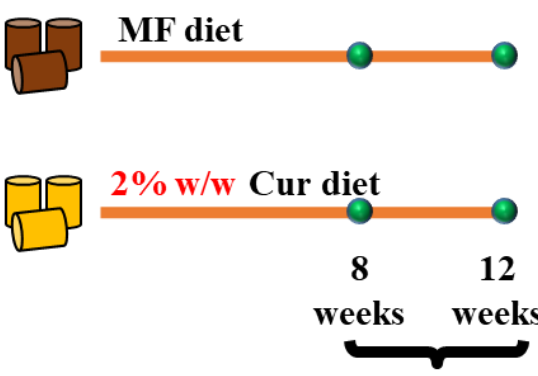

analysis

\section{Amyloid induced groups}

AApoAII fibrils

$1 \mu \mathrm{g} / \mathrm{mouse}$
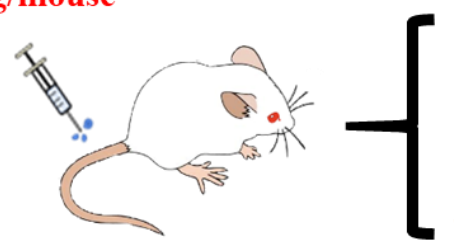

A-NT (Induction)

R1.P1-Apoa $2^{c}$ congenic mice (female, 8-week-old)
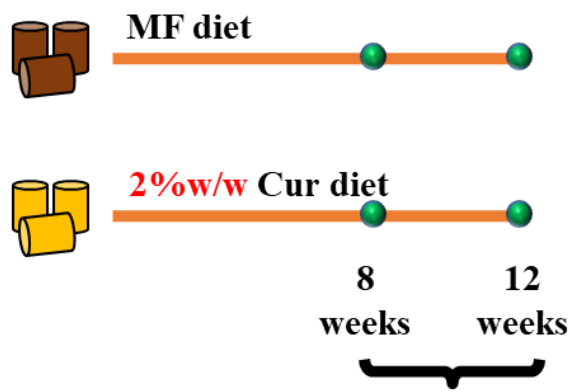

analysis

Eight-week-old female R1.P1-Apoa2c mice were divided into 4 groups: control (Con) group, curcumin (Cur) group, amyloid and no-treatment (A-NT) group, and amyloid and curcumin (A-Cur) group. The A-NT and A-Cur groups were injected with $1 \mu \mathrm{g} /$ mouse of AApoAII fibrils to induce amyloidosis, and the Con and Cur groups were injected with distilled water instead of amyloid fibrils. Mice in the Con and A-NT groups were fed a commercial diet from 8 weeks of age, while mice in the Cur and A-Cur groups were fed a $2 \% \mathrm{w} / \mathrm{w}$ curcumin diet. Mice were sacrificed for biochemical analysis and histochemical analysis after 8 weeks or 12 weeks $(\mathrm{N}=$ 3 - 5 in each group). 


\section{Supplementary Figure 2}

a

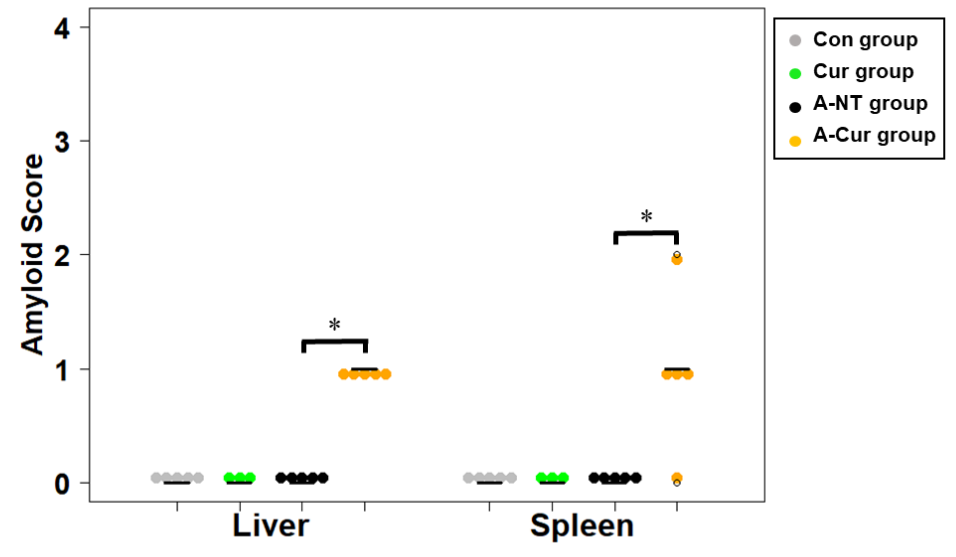

1

b

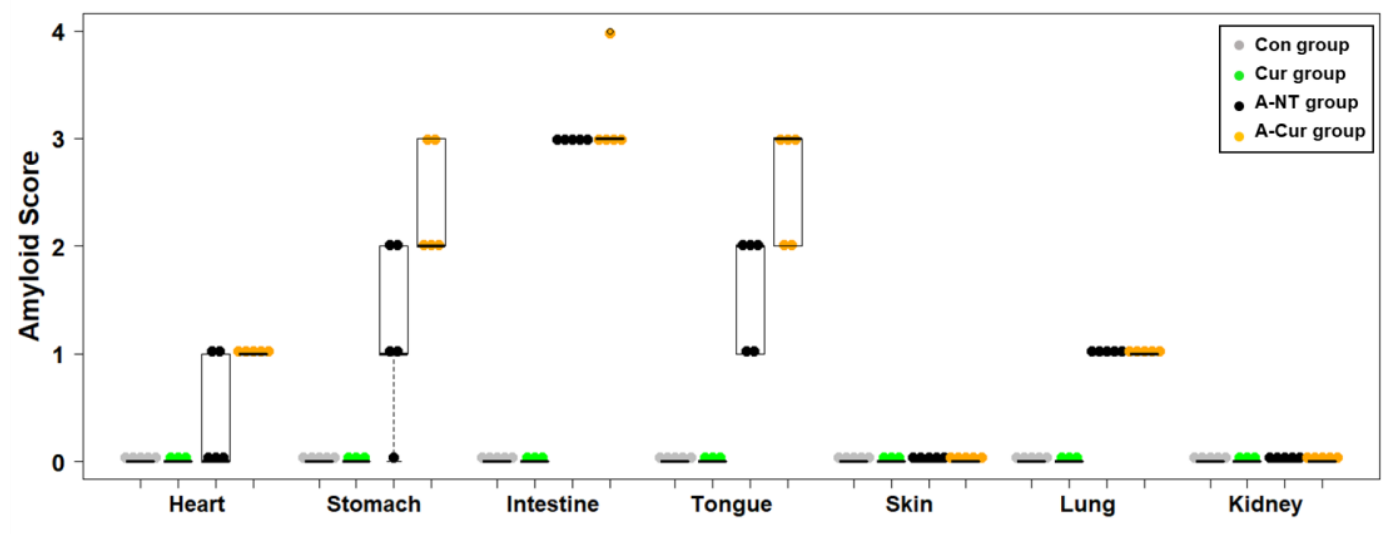

2

C

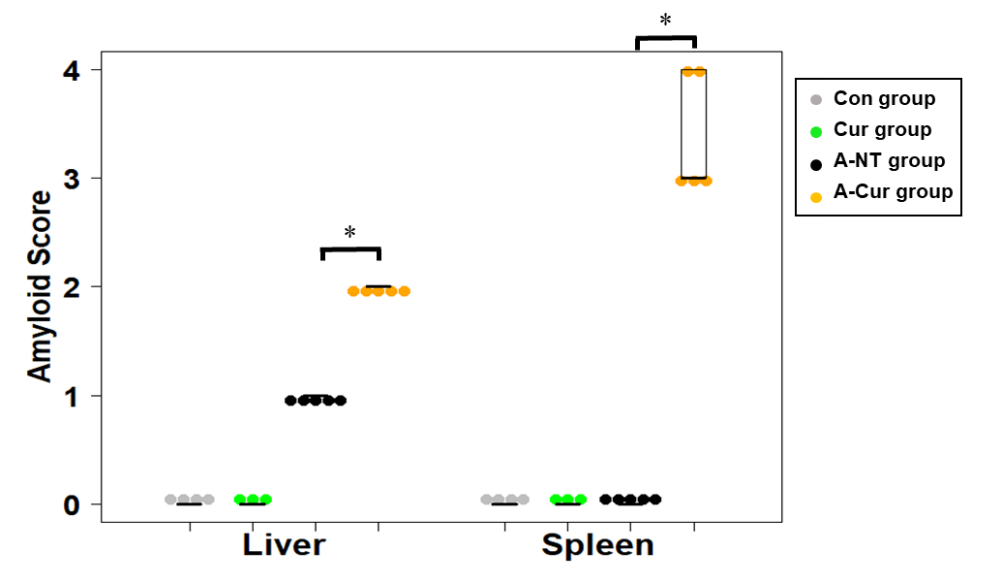




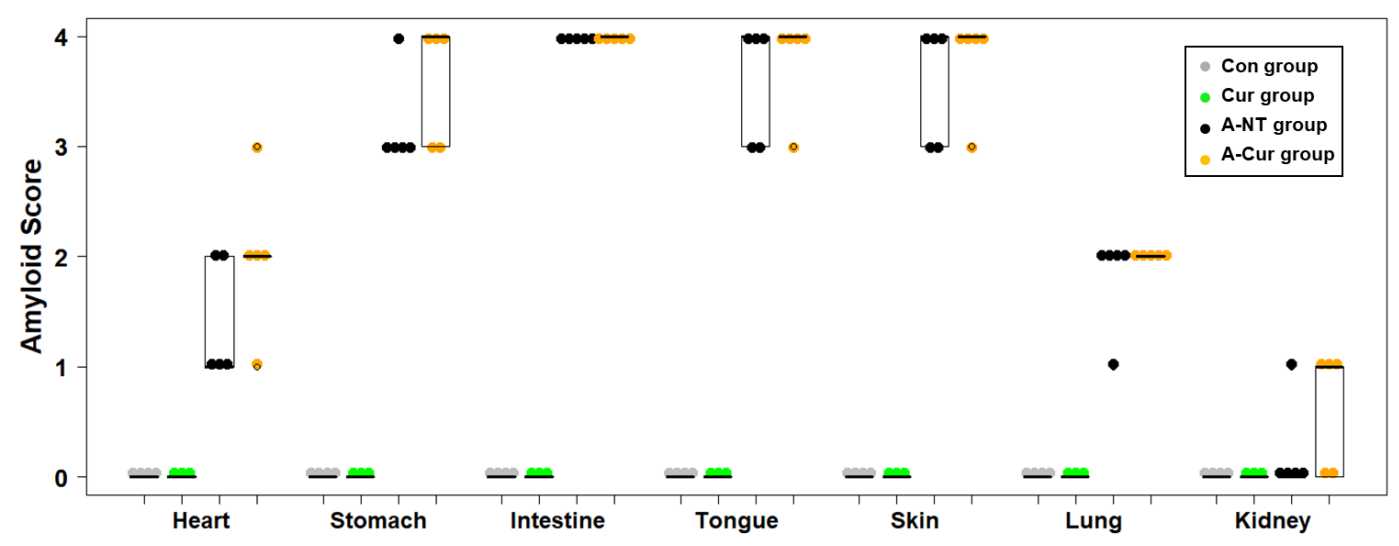

Supplementary Figure 2. Amyloid score in various organs

(a) Amyloid deposition in the liver and spleen is increased by supplementation with curcumin for 8 weeks. (b) Amyloid score in other organs (heart, stomach, intestine, tongue, skin, lung, kidney) in the 8-week group. (c) Amyloid deposition in the liver and spleen is increased by supplementation with curcumin for 12 weeks. (d) Amyloid score in other organs (heart, stomach, intestine, tongue, skin, lung, kidney) in the 12-week group. Each dot represents an individual mouse. The Kruskal-Wallis test with the Steel-Dwass test was used for comparisons of the amyloid score; $* \mathrm{P}<0.05$.

Supplementary Table 1 Liver and Body Weight

\begin{tabular}{|c|c|c|c|}
\hline 2\%w/w Cur exp. & Body weight (g) & Liver weight (g) & Liver/body weight 9 \\
\hline Con (8-week group) & $27.178 \pm 2.604$ & $1.249 \pm 0.085$ & $0.0461 \pm 0.00210$ \\
\hline Cur (8-week group) & $25.965 \pm 1.107$ & $1.573 \pm 0.079$ & $0.0606 \pm 0.00111$ \\
\hline A-NT (8-week group) & $25.354 \pm 1.842$ & $1.254 \pm 0.111$ & $0.0496 \pm 0.00512$ \\
\hline A-Cur (8-week group) & $26.804 \pm 1.728$ & $1.458 \pm 0.124$ & $0.0544 \pm 0.00313$ \\
\hline Con (12-week group) & $25.044 \pm 1.729$ & $1.140 \pm 0.141$ & $0.0455 \pm 0.00314$ \\
\hline Cur (12-week group) & $23.570 \pm 0.182$ & $1.443 \pm 0.161$ & $0.0612 \pm 0.00715$ \\
\hline A-NT (12-week group) & $24.878 \pm 2.044$ & $1.217 \pm 0.147$ & $0.0488 \pm 0.00316$ \\
\hline A-Cur (12-week group) & $26.295 \pm 2.881$ & $1.872 \pm 0.162$ & $0.0714 \pm 0.00417$ \\
\hline
\end{tabular}


bioRxiv preprint doi: https://doi.org/10.1101/2020.09.23.309302; this version posted September 23, 2020. The copyright holder for this

preprint (which was not certified by peer review) is the author/funder, who has granted bioRxiv a license to display the preprint in perpetuity. It is made available under aCC-BY-NC-ND 4.0 International license.

Supplementary Figure 3

a
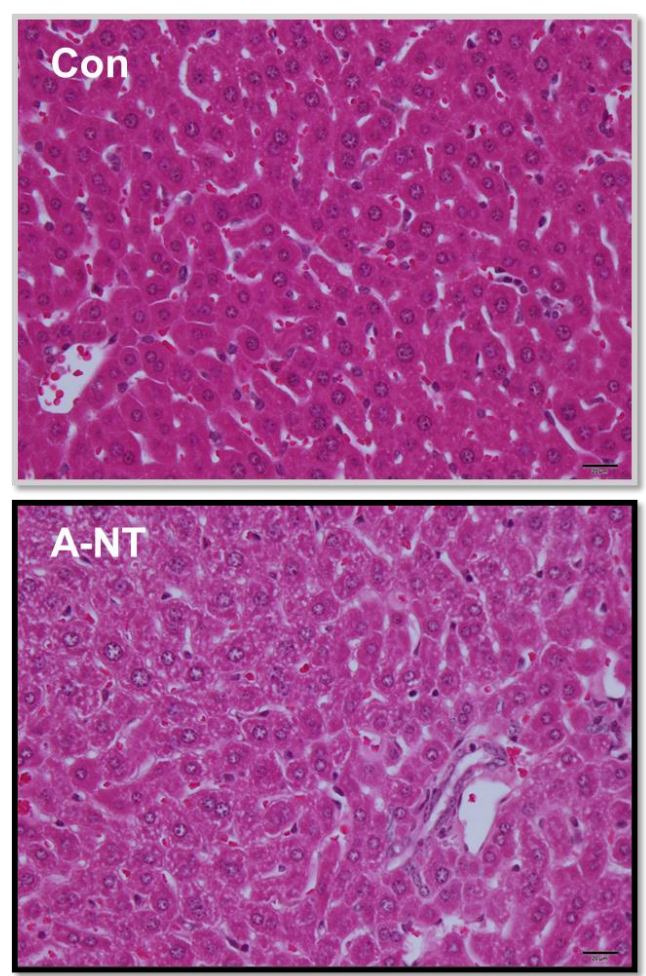
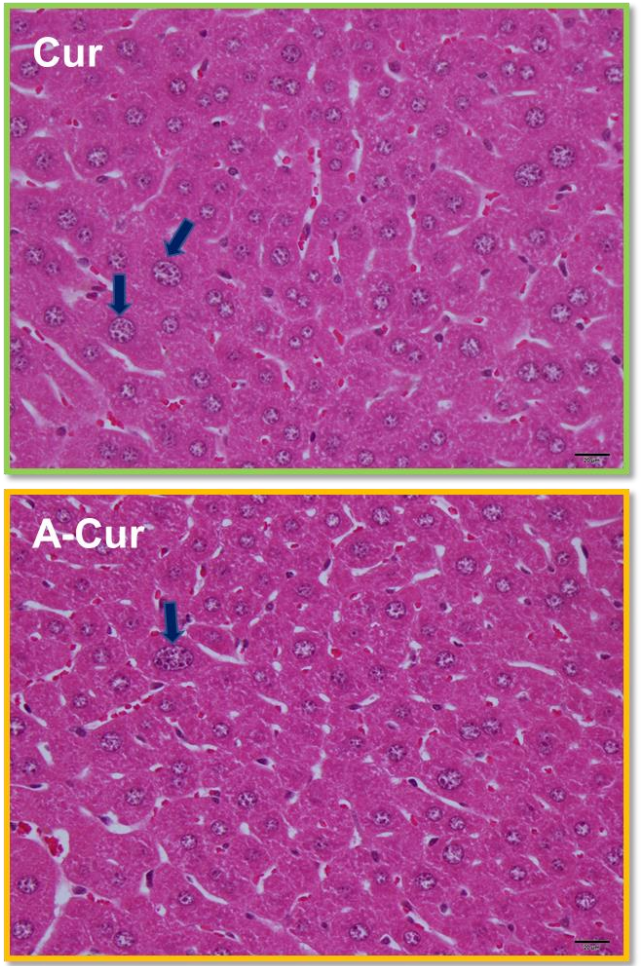

2

b

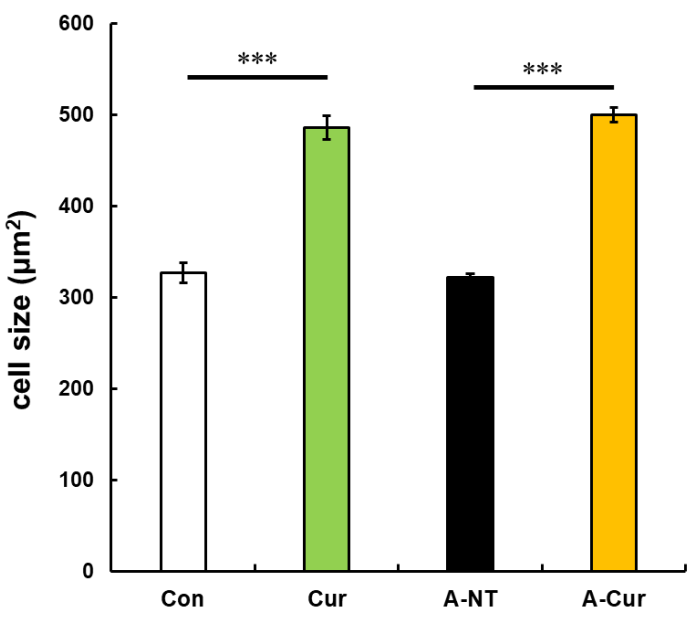

3

\section{Supplementary Figure 3. Hepatocyte hypertrophy in curcumin intake groups}

(a) Representative HE stained image of hepatocytes. Large nuclei (black arrows) were identified in curcumin intake groups. Each scale bar indicates $20 \mu \mathrm{m}$. (b) Average hepatocyte size in each group. Data represent the mean \pm SEM. $\mathrm{N}=3-5$. The Tukey-Kramer method was used for multiple comparisons of hepatocyte size; $* * *$ $\mathrm{P}<0.001$. 
2

\section{a}

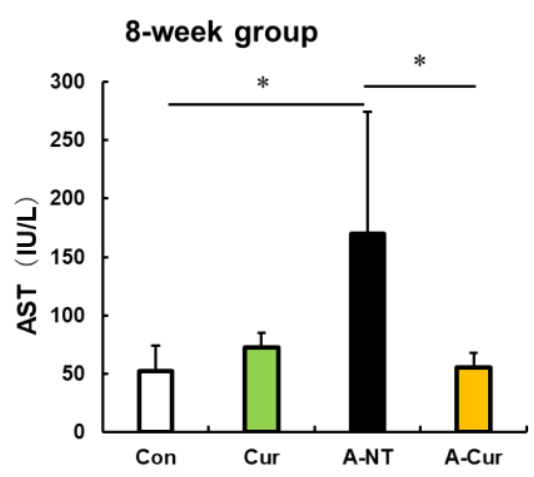

b

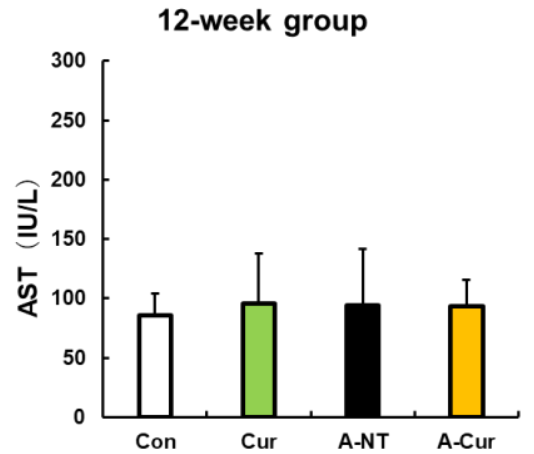

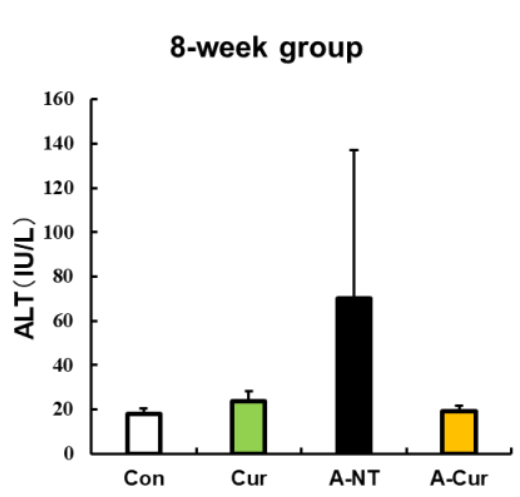

12-week group

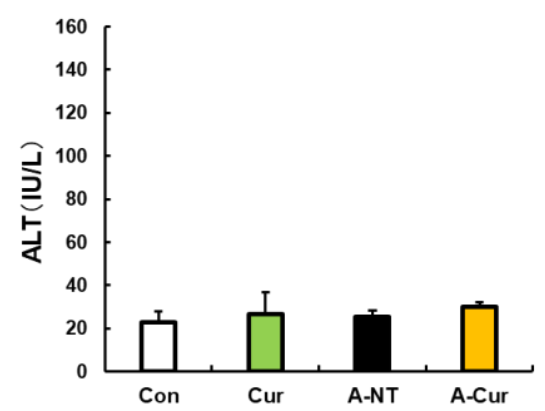

Supplementary Figure 4. No differences in AST and ALT plasma concentrations after curcumin intake. AST and ALT plasma levels were detected to evaluate hepatocyte injury. (a) AST and ALT concentrations in the 8-week group. (b) AST and ALT plasma concentrations in the 12-week group. Data are mean $\pm \mathrm{SD} . \mathrm{N}=3-5$. The Tukey-Kramer method was used for multiple comparisons; $* \mathrm{P}<0.05$.

\section{Supplementary Figure 5}

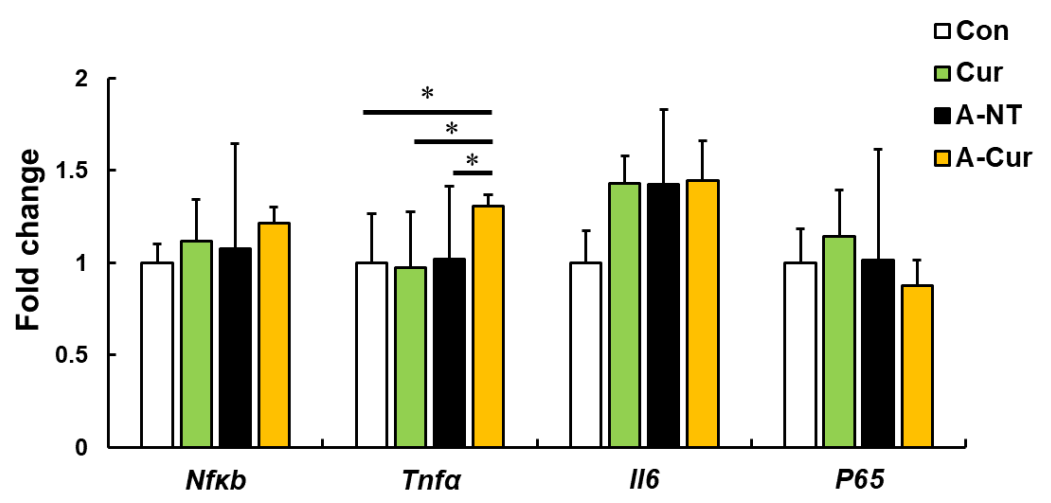

Supplementary Figure 5. Inflammation-related gene expression in the liver in the 12-week group.

Representative genes related to inflammation were measured by real-time qPCR. Results show that curcumin does not affect the expression of inflammatory genes, but Tnfa was increased in the A-Cur group, in which mice exhibit severe amyloid deposition. Data are mean $\pm \mathrm{SD} . \mathrm{N}=3-5$. The Tukey-Kramer method was used for multiple comparisons; $* \mathrm{P}<0.05$. 


\section{Supplementary Figure 6}

2

3

a

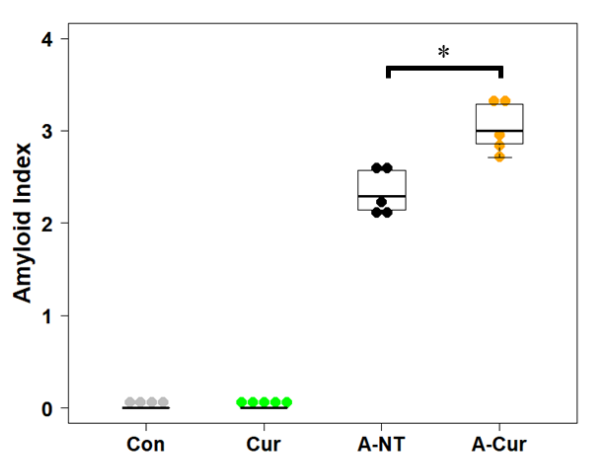

b

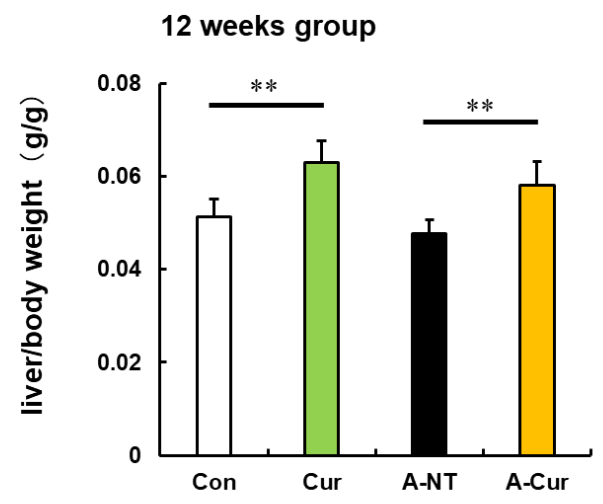

Supplementary Figure 6. Degree of AApoAII amyloid deposition and liver weights were significantly increased after supplementation with low dose curcumin diet $(0.5 \% \mathrm{w} / \mathrm{w})$.

(a) Amyloid index (AI) in the 12-week group supplemented with $0.5 \%$ curcumin diet. Each dot represents an individual mouse. (b) ratio of liver weight / body weight in the 12-week group. Data are mean $\pm \mathrm{SD} . \mathrm{N}=4-5$. The Kruskal-Wallis test with the Steel-Dwass test was used for the amyloid index, and the Tukey-Kramer method was used for multiple comparisons of liver weights; $* \mathrm{P}<0.05, * * \mathrm{P}<0.01$.

\section{Supplementary Figure 7}

a
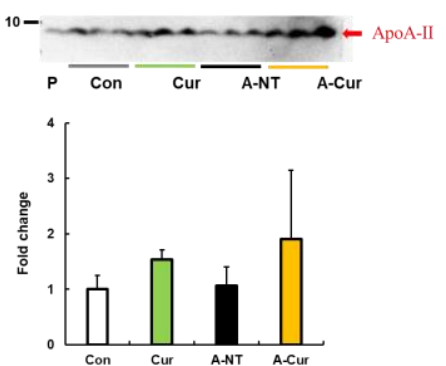

b

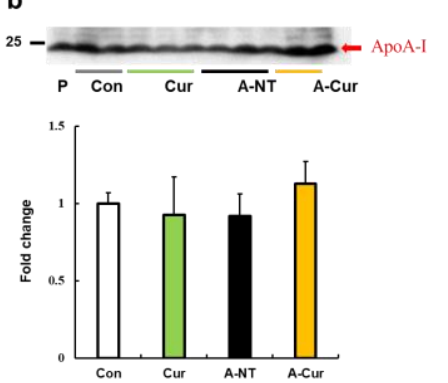

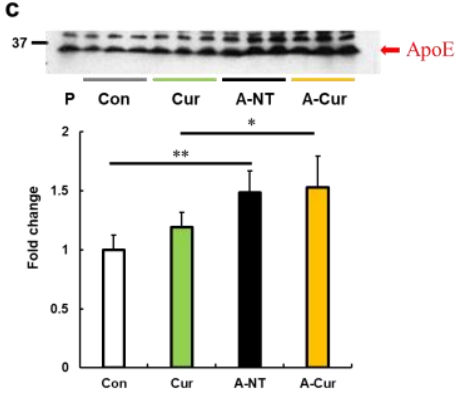

Supplementary Figure 7. ApoA-II, ApoA-I and ApoE plasma levels after supplementation with $2 \%$ w/w curcumin diet for 8 weeks.

(a-c) Plasma levels of ApoA-II, ApoA-I, and ApoE proteins were determined by Western immunoblot. There were no obvious changes in ApoA-II and ApoA-I plasma concentrations; the concentration of ApoE increased significantly in the amyloid-induced groups. Histograms show fold changes relative to the Con group and represent the means $\pm \mathrm{S}$ D. P indicates the pooled plasma of female R1.P1-Apoa2c mice at 2 months of age (N $=4$ ) that did not have AApoAII amyloid deposits, as the positive control of these proteins. $\mathrm{N}=3-5$. The Tukey-Kramer method was used for multiple comparisons; $* \mathrm{P}<0.05, * * \mathrm{P}<0.01$. 
bioRxiv preprint doi: https://doi org/10.1101/2020.09.23.309302; this version posted September 23, 2020. The copyright holder for this preprint (which was not certified by peer review) is the author/funder, who has granted bioRxiv a license to display the preprint in perpetuity. It is made available under aCC-BY-NC-ND 4.0 International license.

$1 \quad$ Supplementary Figure 8

Ppary in liver

2

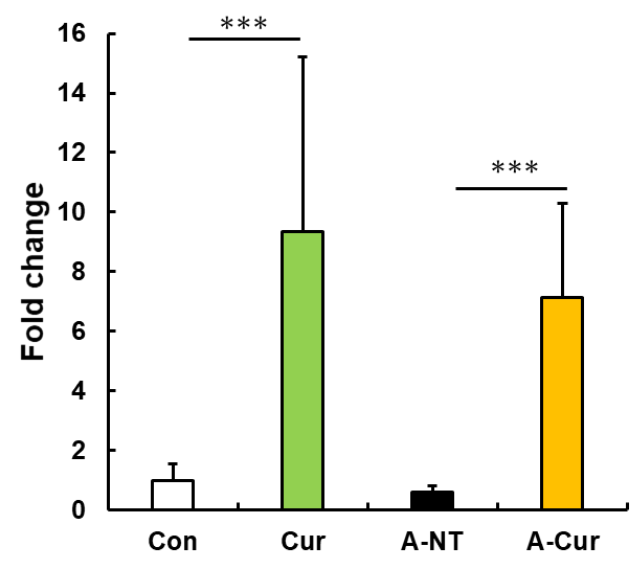

Supplementary Figure 8. Ppary in the liver

Ppary expression in the liver is upregulated in curcumin intake groups. Histograms show fold changes relative to the Con group. Data are mean $\pm \mathrm{SD}$. The Tukey-Kramer method was used for multiple comparisons; ${ }^{* * *} \mathrm{P}<$ 0.001 .

\section{Supplementary Figure 9}

a

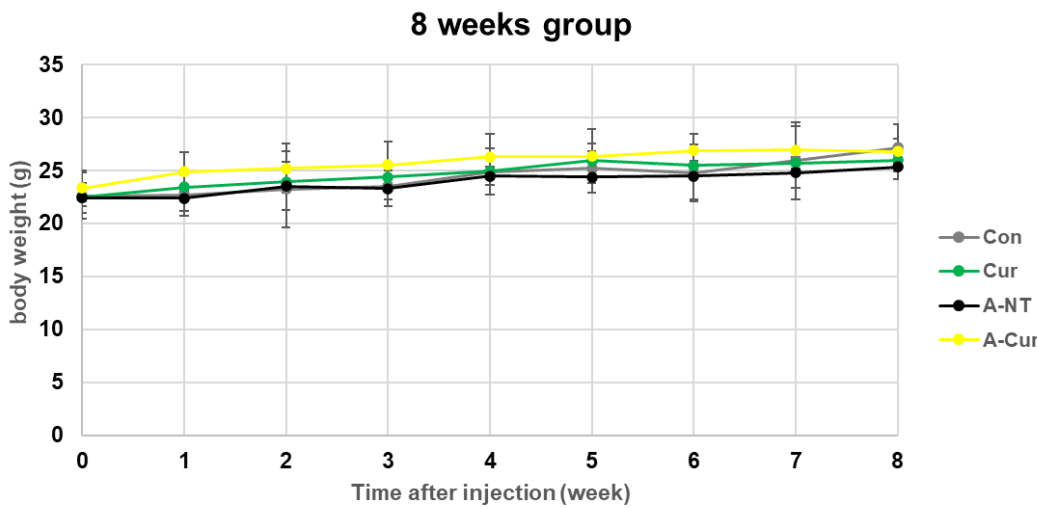

b

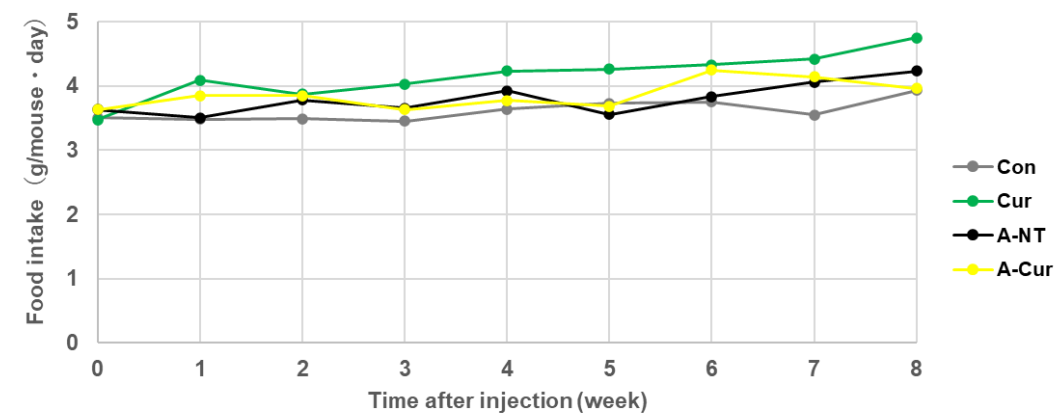


1

2

3

4

5

6

7

8

9

10

11

13

14

15

16

17

18

19

20

21

22

23

24

25

26

27

28

29

30

31

32

33
C

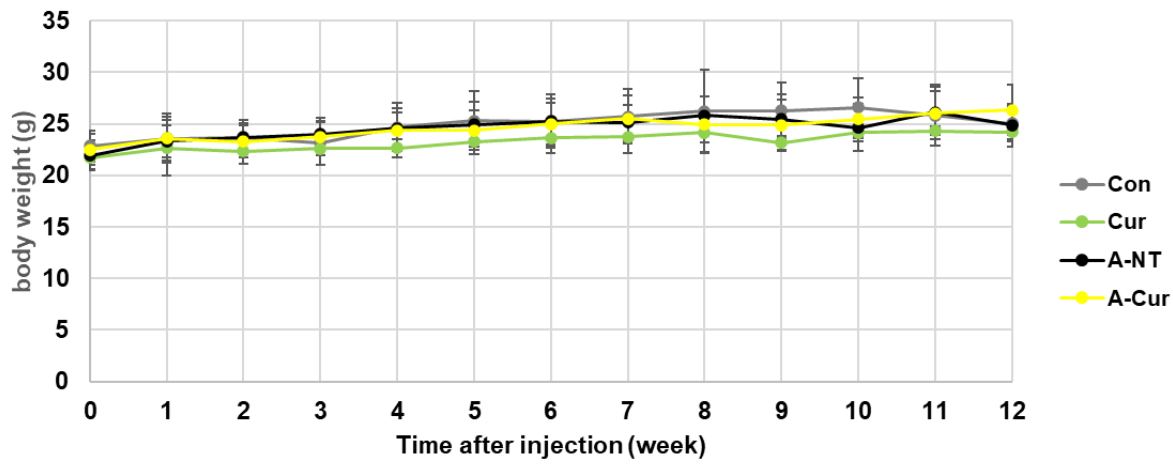

d

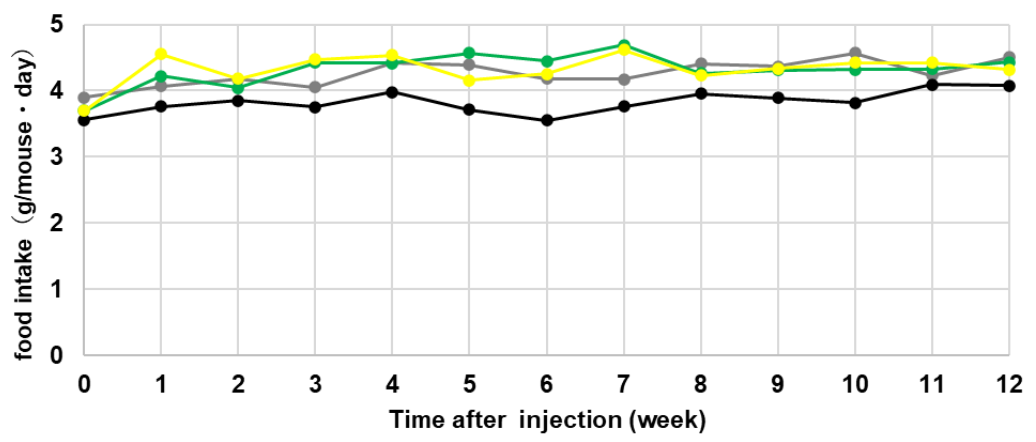

12 weeks group

$\rightarrow-$ Con

$\rightarrow-$ Cur

$\rightarrow$ A-NT

$=$ A-Cur

Supplementary Figure 9. Weekly body weight and food intake measurements. (a) Body weight and (b) food intake in the 8 -week group. Each column and bar represent the mean \pm S.D. $(\mathrm{N}=3-5$ ). (c) Body weight and (d) food intake in the 12-week group. Data are represented in $\mathrm{g} /$ mouse $\cdot$ day (food intake). 
Supplementary Table 4. Specific primers used in real-time qPCR.

\begin{tabular}{|l|l|l|}
\hline & Foward & Reverse \\
\hline$\beta$-actin & ACAATGAGCTGCGTGTGGCC & CCTCGTAGATGGGCACAGTG \\
\hline Apoa1 & GTGGCTCTGGTCTTCCTGAC & ACGGTTGAACCCAGAGTGTC \\
\hline Apoa2 & GCCTGTTCACTCAATACTTTCAG & CAGACTAGTTCCTGCTGACC \\
\hline Ppara & GCGTACGGCAATGGCTTTATC & GTTTAGAAGGCCAGGCCGATC \\
\hline Ppary & CCACCAACTTCGGAATCAGCT & GTATTCTTGGAGCTTCAGGTC \\
\hline Nfkb & ATGGCAGACGATGATCCCTAC & TGTTGACAGTGGTATTTCTGGTG \\
\hline Tnfa & ACGGCATGGATCTCAAAGAC & AGATAGCAAATCGGCTGACG \\
\hline Il6 & CCCAATTTCCAATGCTCTCC & TGAATTGGATGGTCTTGGTCC \\
\hline P65 & GCTATAACTCGCCTGGTGAC & CTGTCAGCACCTTAGGAGC \\
\hline Cd36 & GTGCTGATCCTTTCAGAGTCTC & CAGCAATGAGCCCACAGTTCC \\
\hline Fabp1 & AGGTCTGCCCGAGGACCTCAT & CCAGTCATGGTCTCCAGTTCG \\
\hline Scd1 & GAGGCCTGTACGGGATCATA & GCCGTGCCTTGTAAGTTCTGTG \\
\hline
\end{tabular}

